
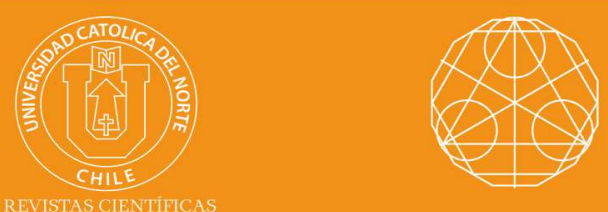

\title{
Existence of solution for some quasilinear parabolic systems with weight and weak monotonicity
}

Azroul Elhoussine ${ }^{1}$ (1) orcid.org/0000-0002-2396-4844

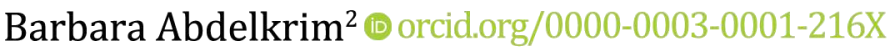

Rami El Houcine ${ }^{3}$ (1) orcid.org/0000-0003-4087-9104

Sidi Mohammed Ben Abdellah University, Dept. of Mathematics, Laboratory LAMA, Fez, Morocco.

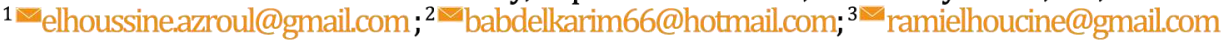
Received: January 2019 | Accepted: January 2020

\section{Abstract:}

We prove the existence of weak solution u for the nonlinear parabolic systems:

$$
(Q P S)_{(\omega)}\left\{\begin{aligned}
\partial_{t} u-\operatorname{div} \sigma(x, t, u, D u) & =v(x, t)+f(x, t, u)+\operatorname{divg}(x, t, u) \text { in } \Omega_{T} \\
u(x, t) & =0 \text { on } \partial \Omega \times(0, T) \\
u(x, 0) & =u_{0}(x) \text { on } \Omega
\end{aligned}\right.
$$

which is a Dirichlet Problem. In this system, $v$ belongs to $L^{p^{\prime}}\left(0, T, W^{-1, p^{\prime}}\left(\Omega, \omega^{*}, \mathbf{R}^{m}\right)\right)$; and $u_{0} \in L^{2}\left(\Omega, \omega_{0}, \mathbf{R}^{m}\right)$, $f$ and $g$ satisfy some standards continuity and growth conditions. We prove existence of a weak solution of different variants of this system under classical regularity for some $p \in] \frac{2 n}{n+2} ; \infty[$, growth and coercivity for $\sigma$ but with only very mild monotonicity assumptions.

Keywords: Nonlinear paraboliic system; Young measure; The divcurl type inequality.

\section{MSC (2010): 54A05, 54A10, 54C05.}

\section{Cite this article as (IEEE citation style)}

A. Elhoussine, A. Barbara, and R. El Houcine, "Existence of solution for some quasilinear parabolic systems with weight and weak monotonicity", Proyecciones (Antofagasta, On line), vol. 39, no. 3, pp. 529-557, Jun. 2020, doi: 10.22199/issn.0717-6279 2020-03-0033.

Article copyright: (C) 2020 Azroul Elhoussine, Barbara Abdelkrim and Rami El Houcine. This is an open access article distributed under the terms of the Creative Commons Licence, which permits unrestricted use and distribution provided the original author and source are credited

(cc) BY 


\section{Introduction}

Let $\Omega$ be a bounded open domain in $\mathbf{R}^{n}, n \geq 2$ and $0<T<\infty$ is given; we recall that $\Omega_{T}$ is defined as the cylinder $\Omega \times(0 ; T)$ this part is devoted to establish Leray-lions existence results for parabolic problems in divergence form of type $(Q P S)_{(\omega)}$. In this paper, the aims of this text is to prove existence results under relaxed monotonicity, in particular under strict quasi-monotonicity. The main technical tool we advocate and use throughout the proof are Young measures. By applying a Galerkin schema, we obtain easily an approximating sequence $u_{k}$. The Ball's theorem and especially the resulting tools mode available by Hungerbühler [9] to partial differential equation theory give then a sufficient control on the gradient approximating sequence $D u_{k}$ to pass to the limit. This method is used by Dolzmann, Muller and Mainly by Hungurbühler to get the existence of a weak solution for the quasi-linear elliptic system and quasi-linear parabolic. Among the authors who have studied the existence of a solution of parabolic equations, we find Dominique Blandand and Redwane Hicham [5]. This paper can be seen as generalization of Hungurbühler. This problem was made by, Hungerbühler N, see [9] in a space of sobolev without weight, with $p \in] \frac{2 n}{n+2} ; \infty[$.

\section{2. functional framework}

Definition 2.1. : Let $X$ be a Banach space and $0<T<\infty$, the space $L^{p}(0, T, X)$ with $1 \leq p \leq \infty$ consists of all measurable functions (identified modulo the relation "equal almost everywhere") $u:(0 ; T) \rightarrow X$ for which

$$
\begin{gathered}
\|u\|_{L^{p}(0, T, X)}=\left(\int_{0}^{T}\|u(t)\|_{X}^{p} d t\right)^{\frac{1}{p}}, \text { if } p \geq 1 ; \\
\|u\|_{L^{p}(0, T, X)}=e s s \sup _{t \in(0 ; T)}\|u(t)\|_{X}, \text { if } p=\infty \text { holds. }
\end{gathered}
$$

Again, equipped with the norm $\|\cdot\|_{L^{p}(0, T, X)} ; L^{p}(0, T, X)$ is a Banach space. In the special case where $p=2$ and $X$ is a Hilbert space $L^{2}(0, T, X)$ equipped with the inner product $\langle u, v\rangle=\int_{0}^{T}\langle u(t), v(t)\rangle_{X} d t$; for all $u ; v \in$ $L^{2}(0, T, X)$ is a Hilbert space, Moreover; if $1 \leq q \leq \infty$ the following Hölder inequality holds for all $v \in L^{p^{\prime}}\left(0, T, X^{\prime}\right)$ and all $u \in L^{p}(0, T, X)$. $\int_{0}^{T}|\langle u(t), v(t)\rangle| d t \leq\|v\|_{L^{p^{\prime}\left(0, T, X^{\prime}\right)}} \cdot\|u\|_{L^{p}(0, T, X)}$ 
and $L^{q}(0, T, X) \hookrightarrow L^{p}(0, T, Y)$, for all $X, Y$ such that $X \hookrightarrow Y$.

$C^{k}([0 ; T], X)$ are dense in $L^{p}(0, T, X)$ as $p \leq \infty$.

$C^{k}([0 ; T], X) \hookrightarrow L^{p}(0, T, X)$ is continuous. If $1 \leq p<\infty$ and $X$ is separable; so is $L^{p}(0, T, X)$. If $1<p<\infty, L^{p}(0, T, X)$ is uniformly convex in the case where $X$ is uniformly convex, and, reflexive, separable when $X$ is reflexive and separable Banach space.

Definition 2.2. (Lebesgues and Sobolev spaces with weight): For $1 \leq p \leq$ $\infty$ and $k \in \mathbf{N}$; we consider the weight spaces:

$$
L^{p}\left(\Omega, \omega_{0}, \mathbf{R}^{m}\right) \equiv\left\{u=u(x) ; u_{j} \omega_{0 j}^{\frac{1}{p}} \in L^{p}\left(\Omega, \mathbf{R}^{m}\right)\right\}
$$

with, $\omega_{0}=\left(\omega_{0 j}\right)_{1 \leq j \leq m}$.

$W^{k, p}\left(\Omega, \omega_{\alpha}, \mathbf{R}^{m}\right) \equiv\left\{u \in L^{p}\left(\Omega, \omega_{\alpha}, \mathbf{R}^{m}\right) ; \quad D^{\alpha} u \in L^{p}\left(\Omega, \omega_{\alpha}, \mathbf{R}^{m}\right) ;\right.$ for $\left.0 \leq|\alpha| \leq k\right\}$.

$D^{\alpha} u$ is the weak derivative of $u$.

The weighted functions $\omega_{\alpha}$ defined by:

$$
\omega_{\alpha}=\left\{\begin{array}{r}
\omega_{0}=\left(\omega_{0 j}\right)_{1 \leq j \leq m} \text { if }|\alpha|=0, \\
\omega=\left(\omega_{i j}\right)_{1 \leq i \leq n ; 1 \leq j \leq m} \text { if }|\alpha|=1, \\
\text { arbitrary weight functions } \quad \text { if } 2 \leq|\alpha| \leq k .
\end{array}\right.
$$

And $\omega_{i j}^{*}=w_{i j}^{1-p^{\prime}}$ is the conjugate of $\omega_{i j}$. We defined the inner product in $L^{2}\left(\Omega, \omega_{0}, \mathbf{R}^{m}\right)$ by: $\langle u, v\rangle_{2, \omega_{0}}=\sum_{j=1}^{m} \int_{\Omega}\left(\left(w_{0 j}\right)^{\frac{1}{2}} u_{j}\right)\left(\left(w_{0 j}\right)^{\frac{1}{2}} v_{j}\right) d x$,

for all $u ; v$ in $L^{2}\left(\Omega, \omega_{0}, \mathbf{R}^{m}\right)$. And for all $u ; v$ in $W_{0}^{r, 2}\left(\Omega, \omega_{\alpha}, \mathbf{R}^{m}\right)$ we denotes the inner product by:

$$
\begin{gathered}
\langle u, v\rangle_{W^{r, 2}\left(\omega_{\alpha}\right)}=\langle u, v\rangle_{2, \omega_{0}}+\sum_{i ; j=1}^{n ; m} \int_{\Omega}\left(w_{i j}^{\frac{1}{2}} D_{i j} u\right)\left(w_{i j}^{\frac{1}{2}} D_{i j} v\right) d x \\
+\sum_{2 \leq|\alpha| \leq r} \int_{\Omega}\left(w_{\alpha}^{\frac{1}{2}} D^{\alpha} u\right)\left(w_{\alpha}^{\frac{1}{2}} D^{\alpha} v\right) d x .
\end{gathered}
$$

We denote $W_{0}^{k, p}\left(\Omega, \omega_{\alpha}, \mathbf{R}^{m}\right)$ the closure of $C_{0}^{\infty}\left(\Omega, \mathbf{R}^{m}\right)$ in $W^{k, p}\left(\Omega, \omega_{\alpha}, \mathbf{R}^{m}\right)$, where the norm is $\|\cdot\|_{k, p, \omega_{\alpha}}$ is given by: 
$\|u\|_{k, p, \omega_{\alpha}}=\left(\sum_{0 \leq|\alpha| \leq k}\left\|D^{\alpha} u\right\|_{p} \omega_{\alpha}\right)^{\frac{1}{p}}$.

We have:

$$
W_{0}^{k, p}\left(\Omega, \omega_{\alpha}, \mathbf{R}^{m}\right) \hookrightarrow W^{k, p}\left(\Omega, \omega_{\alpha}, \mathbf{R}^{m}\right) \hookrightarrow L^{p}\left(\Omega, \omega_{0}, \mathbf{R}^{m}\right) .
$$

$\left(W_{0}^{k, p}\left(\Omega, \omega_{\alpha}, \mathbf{R}^{m}\right)\right)^{\prime} \equiv W^{-k, p^{\prime}}\left(\Omega, \omega_{\alpha}^{*}, \mathbf{R}^{m}\right)$.

$\mathbf{M}^{m \times n}$ denotes the space of real $m \times n$ matrices equipped with inner product $M: N=M_{i j} N_{i j}$ (we use the usual summation convention) and the tensor product $a \otimes b$ of two vectors $a, b \in \mathbf{R}^{n}$ is defined to be the matrix $\left(a_{i} b_{j}\right)_{i, j=1, \ldots, m}$.

Definition 2.3. let $\Omega$ be a measurable subset of $\mathbf{R}^{n},\left(\mu_{k}\right)_{k \in \mathbf{N}}$ and $\mu$ be a sequence and respectively an element in $M\left(\Omega, \mathbf{R}^{m}\right)$. we say that $\left(\mu_{k}\right)$ converges weakly $*$ to $\mu$, which is denoted by $\mu_{k} \rightarrow^{*} \mu$, if:

$$
\int_{\Omega} f d \mu_{k} \rightarrow \int_{\Omega} f d \mu, \text { for all } f \in C_{c}\left(\Omega, \mathbf{R}^{m}\right) .
$$

Theorem 2.1. (Ball) Let $\Omega$ be Lebesgue measurable of $\mathbf{R}^{n}$, let $k \subset \mathbf{R}^{m}$ be closed, and let

$u_{j}: \Omega \rightarrow \mathbf{R}^{m}, j \in \mathbf{N}$, be a sequence of Lebesgue measurable functions satisfying $u_{j} \rightarrow K$ in measure as $j \rightarrow \infty$, i.e: given any open neighborhood $U$ of $k$ in $\mathbf{R}^{m}$ such that:

$\lim _{j \rightarrow \infty}\left|\left\{x \in \Omega: u_{j}(x) \notin U\right\}\right|=0$.

Then there exist a sequences $u_{k}$ of $u_{j}$ and a family $\vartheta_{x}$, of positive measure on $\mathbf{R}^{m}$, depending measurably on $x$ such that:

- i) $\left\|\vartheta_{x}\right\|=\int_{\Omega} d \vartheta_{x} \leq 1$, for a.e. $x \in \Omega$.

- ii) $\operatorname{Supp} \vartheta_{x} \subset K$, for a.e. $x \in \Omega$.

- iii) $f\left(u_{k}\right) \rightarrow^{*}\left\langle\vartheta_{x}, f\right\rangle=\int_{\mathbf{R}^{n}} f(\lambda) d \vartheta_{x}(\lambda)$, in $L^{\infty}(\Omega)$; for each $f$ continuous functions

$f: \mathbf{R}^{n} \rightarrow \mathbf{R}$ satisfying $\lim _{|\lambda| \rightarrow \infty} f(\lambda)=0$.

Lemma 2.1. (Fatou) Let $F: \Omega \times \mathbf{R}^{m} \times \mathbf{M}^{m \times n} \rightarrow \mathbf{R}$ be a carathéodory functions and $u_{k}: \Omega \rightarrow \mathbf{R}^{m}$ a sequence of measurable functions such that $D u_{k}$ generates the Young measure $\vartheta_{x}$ with $\left\|\vartheta_{x}\right\|=1$ for almost every 
$x \in \Omega$. Then:

$$
\liminf _{k \rightarrow \infty} \int_{\Omega} F\left(x, u_{k}, D u_{k}\right) d x \geq \int_{\Omega} \int_{\mathbf{M}^{m \times n}} F(x, u, \xi) d \vartheta_{x}(\xi) d x
$$

Provided that the negation $F^{-}\left(x, u_{k}, D u_{k}\right)$ is equiintegrable. More general versions of the this lemma may be found in [13], [14] and [10], proof see [8] ( lemma 1-5).

\section{Hypothesis}

We assume that:

$$
\begin{gathered}
w_{i j} \in L^{1}(\Omega), \quad w_{i j}^{\frac{-1}{p-1}} \in L_{l o c}^{1}(\Omega) \\
w_{i j}^{-s} \in L^{1}(\Omega)
\end{gathered}
$$

for some $s \in] \max \left(\frac{1}{p-1} ; \frac{n}{p}\right) ; \infty\left[\right.$. The Sobolev space $W^{1, p}\left(\Omega, \omega, \mathbf{R}^{m}\right)$ equipped with the norm

$$
\|u\|_{1, p, w}^{p}=\sum_{j=1}^{m} \int_{\Omega} w_{0 j}\left|u_{j}\right|^{p} d x+\sum_{i, j} \int_{\Omega} w_{i j}\left|D_{i j} u\right|^{p} d x .
$$

is a Banach space, reflexive, and $C_{0}^{\infty}\left(\Omega, \mathbf{R}^{m}\right)$ is a subspace of $\left(W^{1, p}\left(\Omega, \omega, \mathbf{R}^{m}\right)\right.$. The space $\left(W_{0}^{1, p}\left(\Omega, \omega, \mathbf{R}^{m}\right)\right.$ is the fermenter of $C_{0}^{\infty}\left(\Omega, \mathbf{R}^{m}\right)$ in $\left(W^{1, p}\left(\Omega, \omega, \mathbf{R}^{m}\right)\right.$ for the norm $\left.\|\cdot\|_{1, p, \omega}^{p}\right)$,

(by the condition 3.1). The norm $\|. .\|_{1, p, w}^{p}$ equivalent to the norm $\||u|\|_{W_{0}^{1, p}\left(\Omega, \omega, \mathbf{R}^{m}\right)}=\left(\sum_{i, j} \int_{\Omega} w_{i j}\left|D_{i j} u\right|^{p} d x\right)^{\frac{1}{p}}$, with $D_{i j} u=\frac{\partial u^{j}}{\partial x_{i}}$ (by: The condition 3.2). If we suppose $\omega_{0 j}=1$, for all $j=1 ; 2 ; \ldots \ldots ; m$, and The condition 3.2, we give:

$$
\left(W ^ { 1 , p } ( \Omega , \omega , \mathbf { R } ^ { m } ) \hookrightarrow \left(W^{1, p_{s}}\left(\Omega, \mathbf{R}^{m}\right) \hookrightarrow L^{r}\left(\Omega, \mathbf{R}^{m}\right),\right.\right.
$$

for all $1 \leq r<p_{s}^{*}$ if $p . s \leq n(s+1)$, and $\forall r \geq 1$ if $p . s>n(s+1)$ with $p_{s}=\frac{p . s}{s+1}$ and $p_{s}^{*}=\frac{n . p . s}{n(s+1)-p . s}$ The Hardy-Type inequalities in condition $\left(P_{0}\right)$ is verifies for $\gamma_{j}=1 ; j=1 ; 2 ; \ldots \ldots ; m$ 
$\left.\left(P_{1}\right) \sigma: \Omega \times\right] 0, T\left[\times \mathbf{R}^{m} \times \mathbf{M}^{m \times n} \longrightarrow \mathbf{M}^{m \times n}\right.$ is a Carathéodory functions, i.e:

$(x, t) \longmapsto \sigma(x, t, u, F)$ is measurable for every $(u, F) \in \mathbf{R}^{m} \times \mathbf{M}^{m \times n}$ and

$(u, F) \in \mathbf{R}^{m} \times \mathbf{M}^{m \times n} \longmapsto \sigma(x, t, u, F)$ is continuous for almost every $(x, t) \in \Omega_{T}$.

$\left(P_{2}\right)$ (Coercivity and growth): There exist constants $c_{1}, c_{2}, \beta>0$ and $\lambda_{1} \in L^{p^{\prime}}\left(\Omega_{T}, \mathbf{R}^{m}\right), \lambda_{2} \in L^{1}\left(\Omega_{T}, \mathbf{R}^{m}\right), \lambda_{3} \in L^{\left(\frac{p}{\alpha}\right)^{\prime}}\left(\Omega_{T}\right), 0<\alpha<p$, $1<q<\infty$, such that, for all $1 \leq r \leq n$, and $1 \leq s \leq m$ :

$$
\begin{gathered}
\left|\sigma_{r s}(x, t, u, F)\right| \leq \beta \omega_{r s}^{\frac{1}{p}}\left[\lambda_{1}+c_{1} \sum_{j=1}^{m} \gamma_{j}^{\frac{1}{p^{\prime}}}\left|u_{j}\right|^{\frac{q}{p^{\prime}}}+c_{1} \sum_{i, j} \omega_{i j}^{\frac{1}{p^{\prime}}}\left|F_{i j}\right|^{p-1}\right] \\
\sigma(x, t, u, F): F \geq-\lambda_{2}(x, t)-\sum_{j=1}^{m} \lambda_{3}(x, t) \omega_{1 j}^{\frac{\alpha}{p}}\left|u_{j}\right|^{\alpha}+c_{2} \sum_{i, j} \omega_{i j}\left|F_{i j}\right|^{p} .
\end{gathered}
$$

$\left(P_{3}\right)$ (Monotonicity): $\sigma$ satisfies one of the following conditions:

- (a) For all $(x, t) \in \Omega_{T}$ and all $u \in \mathbf{R}^{m}$, the map: $F \mapsto \sigma(x, t, u, F)$ is a $C^{1}$ - functions and is monotone.

- (b) There exist a function: $W: \Omega_{T} \times \mathbf{R}^{m} \times \mathbf{M}^{m \times n} \rightarrow \mathbf{R}$, such that $\sigma(x, t, u, F)=\frac{\partial W}{\partial F}(x, t, u, F)$, and $F \mapsto W(x, t, u, F)$ is convex and $C^{1}$ - function.

- (c) For all $(x, t) \in \Omega_{T}$ and all $u \in \mathbf{R}^{m}$, the map: $F \mapsto \sigma(x, t, u, F)$ is strictly monotone .

- (d) $\sigma$ is strictly $-p$ quasi monotone in $F$ i.e: $\int_{\mathbf{M}^{m \times n}}(\sigma(x, t, u, \lambda)-\sigma(x, t, u, \bar{\lambda}):(\lambda-\bar{\lambda}) d \vartheta(\lambda)>0$

$\left(P_{0}\right)$ (The Hardy-Type inequalities); There exist $c>0$, one weighted function $\gamma=\left(\gamma_{j}\right)_{1 \leq j \leq m}$, and the parameter $1<q$, such that:

$$
\left(\sum_{j=1}^{m} \int_{\Omega} \gamma_{j}\left|u_{j}\right|^{q} d x\right)^{\frac{1}{q}} \leq c\left(\sum_{i, j} \int_{\Omega} w_{i j}\left|D_{i j} u\right|^{p} d x\right)^{\frac{1}{p}}
$$

and the expression $\left(\sum_{i, j} \int_{\Omega} w_{i j}\left|D_{i j} u\right|^{p} d x\right)^{\frac{1}{p}}$ is a norm equivalent to the norm $\|\cdot\|_{1 ; p: \omega}$ in $W_{0}^{1, p}\left(\Omega, \omega, \mathbf{R}^{m}\right)$. 
$\left(F_{0}\right)$ (continuity) $f: \Omega_{T} \times \mathbf{R}^{n} \rightarrow \mathbf{R}^{m}$ is a Carathéodory function, i.e. $(x, t) \mapsto$ $f(x, t, u)$ is measurable for every $u \in \mathbf{R}^{n}$ and $u \mapsto f(x, t, u)$ is continuous for almost every $(x, t) \in \Omega_{T}$.

$\left(F_{1}\right)$ (Growth) There exist $0<\gamma<p-1, \quad b_{1} \in L^{p^{\prime}}\left(\Omega_{T}\right)$ and $b_{2} \in$ $L^{\gamma^{\mp}}\left(0, T, L^{\frac{n}{p}}(\Omega)\right)$, such that:

$\left|f_{k}(x, t, u)\right| \leq b_{1} \omega_{0 k}^{\frac{1}{p}}+\sum_{j=1}^{m} b_{2}\left|u_{j}\right|^{\gamma} \omega_{0 j}^{\frac{p-1}{p^{*}}} \omega_{0 k}^{\frac{1}{p^{*}}}: \quad k=1 ; 2 ; \ldots ; m$.

$\gamma^{\mp}=\left(\frac{p}{p-1-\gamma}\right)^{\prime}$ is the Hölder conjugate of $\frac{p}{\gamma+1}$.

$\left(G_{0}\right)$ (continuity) $g: \Omega_{T} \times \mathbf{R}^{n} \rightarrow \mathbf{M}^{m \times n}$ is a Carathéodory function.

$\left(G_{1}\right)$ (Growth) There exist $0<\eta<p-1, \quad b_{4} \in L^{p^{\prime}}\left(\Omega_{T}\right)$ and $b_{5} \in$ $L^{\infty}\left(0, T, L^{\frac{n}{p-1}}(\Omega)\right)$, such that:

$$
\left|g_{r s}(x, t, u)\right| \leq b_{4} \omega_{r s}^{\frac{1}{p}}+\sum_{j=1}^{m} b_{5}\left|u_{j}\right|^{\eta} \omega_{0 j^{\frac{n-p+1}{p^{\prime}}}} \omega_{r s}^{\frac{n-p+1}{n p}}
$$

\section{Remark 3.1.}

$\left(P_{1}\right)$ ( Carathéodory condition) ensures that $\sigma(x, t, u(x, t), U(x, t))$ is measurable on $\partial \Omega_{T}$ for measurable functions:

$u: \Omega_{T} \rightarrow \mathbf{R}^{m}$

$U: \Omega_{T} \rightarrow \mathbf{M}^{m \times n}$.

The growth condition and coercivity

$\left(P_{2}\right)$ is standard it is used in the construction of approximate solutions by a Galerkin method and when we pass to the limit. Although the strict monotonicity condition $(c)$ in $\left(P_{3}\right)$ ensure existence of a weak solution by standard method see e- $g$ [11], the main point is that we do not require strict monotonicity or monotonicity in the variables $(u, F)$ in $(a),(b)$ and $(d)$ as it is usually assumed in previous works see-e-g [3], [11],[1]. Under these mild assumptions and using again Young measures, Hungerbühler shows in [9] that initial and boundary value problem $(\mathcal{Q P S})_{\omega}$ has a weak solution $u \in L^{p}\left(0, T, W_{0}^{1, p}\left(\Omega, \omega, \mathbf{R}^{m}\right)\right.$ in following sense.

Definition 3.1. We say that: $u: \Omega_{T} \rightarrow \mathbf{R}^{m}$ is weak solutions of: $(\mathcal{Q P S})_{\omega}\left\{\begin{aligned} \partial_{t} u-\operatorname{div} \sigma(x, t, u, D u) & =v(x, t)+f(x, t, u)+\operatorname{divg}(x, t, u) \text { in } \Omega_{T} \\ u(x, t) & =0 \text { on } \partial \Omega \times(0, T) \\ u(x, 0) & =u_{0}(x) \text { on } \Omega\end{aligned}\right.$ where $v \in L^{p^{\prime}}\left(0, T, W_{0}^{-1, p^{\prime}}\left(\Omega, \omega^{*}, \mathbf{R}^{m}\right)\right)$ if: 
- (i) u belongs to $L^{1}\left(0, T, W_{0}^{1,1}\left(\Omega, \omega, \mathbf{R}^{m}\right)\right)$.

- (ii) $\sigma(., u(),. D u()$.$) belongs to L^{1}\left(\Omega_{T}, \mathbf{M}^{m \times n}\right)$.

- (iii) The initial condition $u(x, 0)=u_{0}(x)$ on $\Omega$ is fulfilled.

- (iv) The equation:

$\sum_{j} \int_{\Omega_{T}} \partial_{t} u^{j} \cdot \varphi^{j}(x, t) \omega_{0 j} d x d t+\int_{\Omega_{T}} \sigma(x, t, u, D u): D \varphi(x, t) d x d t=\langle v, \varphi\rangle+$ $\int_{\Omega_{T}} f(x, t, u) \cdot \varphi(x, t) d x d t-\int_{\Omega_{T}} g(x, t, u): D \varphi(x, t) d x d t$,

holds for every function $\varphi \in C^{\infty}\left(\overline{\Omega_{T}}, \mathbf{R}^{m}\right)$ which is zero in a neighborhood of $(\partial \Omega \times] 0, T[) \cup(\Omega \times\{0 ; T\})$.

Remark 3.2. - (a) In definition the boundary condition $u=0$ on $(\partial \Omega \times] 0, T[)$ is interpreted in the sense of (i) .

- (b) The condition (iv) is understood in the sense that the Galerkin approximations converge to $u_{0}$ in $L^{2}\left(\Omega, \omega_{0}\right)$.

Theorem 3.1. If $p \in] \frac{2 n}{n+2}, \infty\left[\right.$ and if $\sigma$ satisfies the conditions $\left(P_{1}\right)-\left(P_{3}\right)$ then the parabolic system $(Q P S)_{\omega}$ has a weak solution $u \in L^{p}\left(0, T, W_{0}^{1, p}\left(\Omega, \omega, \mathbf{R}^{m}\right)\right)$ for every $v \in L^{p^{\prime}}\left(0, T, W_{0}^{1, p}\left(\Omega, \omega^{*}, \mathbf{R}^{m}\right)\right)$ every $f$ satisfying $\left(F_{0}\right)-\left(F_{1}\right), g$ satisfying $\left(G_{0}\right)-\left(G_{1}\right)$ and $(n \geq 2)$.

\section{A first parabolic div-curl inequality}

We state here the first set of hypothesis:

- $\left(A_{1}\right)$ : The sequence $\left(u_{k}\right)$ is uniformly bounded in $L^{p}\left(0, T, W_{0}^{1, p}\left(\Omega, \omega, \mathbf{R}^{m}\right)\right)$ for some $p \in] \frac{2 n}{n+2} ; \infty[$ and hence (Bay the Elberlem Suljan theorem: see [2]. A subsequence converges weakly in $L^{p}\left(0, T, W_{0}^{1, p}\left(\Omega, \omega, \mathbf{R}^{m}\right)\right)$ to an element denoted by $u$.

- $\left(A_{2}\right)$ : The sequence $\partial_{t} u_{k}$ is uniformly bounded in $L^{p^{\prime}}\left(0, T,\left(W_{0}^{r, 2}\left(\Omega, \omega, \mathbf{R}^{m}\right)\right)^{\prime}\right)$ for some $2<r<p^{*}$ if $p<n$ and $2<r<\infty$ if $p \geq n$.

- $\left.\left(A_{3}\right): \sigma: \Omega \times\right] 0, T\left[\times \mathbf{R}^{m} \times \mathbf{M}^{m \times n} \longrightarrow \mathbf{M}^{m \times n}\right.$ is a Carathéodory function. 
- $\left(A_{4}\right)$ : The sequence $\sigma_{k}(x, t) \equiv \sigma\left(x, t, u_{k}(x, t), D u_{k}(x, t)\right)$ is uniformly bounded in $L^{p^{\prime}}\left(\Omega_{T}, \omega^{*}, \mathbf{M}^{m \times n}\right)$ and therefore equiintegrable (the equiintegrability follows from, the Hölder inequality).

- $\left(A_{5}\right)$ : The sequence $\left(\sigma_{k}(x, t): D u_{k}\right)^{-}$is equiintegrable.

- $\left(A_{6}\right)$ : There exists a sequence $\left(v_{k}\right)$ such that

$$
\begin{aligned}
& v_{k} \rightarrow u \text { in } L^{p}\left(0, T,\left(W_{0}^{1, p}\left(\Omega, \omega, \mathbf{R}^{m}\right)\right)^{\prime}\right) \text { and } \int_{\Omega_{T}} \sigma_{k}(x, t):\left(D u_{k}-\right. \\
& \left.D v_{k}\right) d x d t \rightarrow 0 ; \quad k \rightarrow \infty .
\end{aligned}
$$

Remark 4.1. Note that the assumption $\left(A_{1}\right)$ and $\left(A_{2}\right)$ ensure a strangle convergence in some Lebesgue spaces and in measure. The idea in order to prove such a compactness is to use Albinos lemma. Technically this is achieved by the followings lemma which is slightly more flexible than e-g. the version in [11] ( chap 1, sect 5-2).

Lemma 4.1. Let $B, B_{0}$ and $B_{1}$ be Banach spaces, $B_{0}$ and $B_{1}$ reflexive. Let $\imath: B_{0} \rightarrow B$ be a compact linear map and:

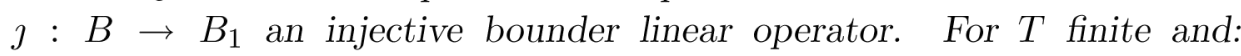
$1<p_{i}<\infty$ with $i=0 ; 1$

$W=\left\{v \in L^{p_{0}}\left(0 ; T ; B_{0}\right): \frac{d}{d t}(\jmath \circ \imath \circ v) \in L^{p_{1}}\left(0 ; T ; B_{1}\right)\right\}$ is a Banach space under the norm:

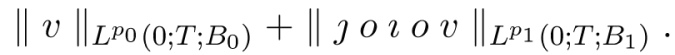

Then, if $V \subset W$ is bounded, the set $\{\imath \quad 0 \quad v ; v \in V\}$ is precompact in $L^{p_{0}}\left(0 ; T ; B_{0}\right)$.

Proof of lemma 4.1 For the proof, see [8] ( Appendix I). For generalization of lemma, see [12]. Now, we apply lemma to obtain the desired bound.

Proposition 4.1. Let $p \in] \frac{2 n}{n+2} ; \infty\left[\right.$ and $\left(u_{k}\right)$ be a sequence satisfying $\left(A_{1}\right)$ and $\left(A_{2}\right)$. Then there exists a subsequence of $\left(u_{k}\right)$ ( for convention not relabeled ) and a functions:

$u \in L^{p}\left(0, T, W_{0}^{1, p}\left(\Omega, \omega, \mathbf{R}^{m}\right)\right)$ such that: $u_{k} \rightarrow u$ in measure on $\Omega_{T}$ and in

$$
L^{p}\left(0, T, L^{r}\left(\Omega, \omega, \mathbf{R}^{m}\right)\right), \forall r<p^{*}
$$

Proof of prop 4.1 Let $\left.\left.B_{0} \equiv W_{0}^{1, p}\left(\Omega, \omega, \mathbf{R}^{m}\right)\right) ; B \equiv L^{r}\left(\Omega, \omega_{0}, \mathbf{R}^{m}\right)\right)$, for same $r$ with: $2<r<p^{*}$, 
and $B_{1} \equiv\left(W_{0}^{r, 2}\left(\Omega, \omega_{\alpha}, \mathbf{R}^{m}\right)\right)^{\prime}$. Since we assumed that $\left.p \in\right] \frac{2 n}{n+2} ; \infty[$, we have the following chain of continuous injections:

$$
\left.B_{0} \hookrightarrow^{i} B \hookrightarrow^{i_{0}} L^{2}\left(\Omega, \omega_{0}, \mathbf{R}^{m}\right)\right) \cong\left(L^{2}\left(\Omega, \omega_{0}, \mathbf{R}^{m}\right)\right)^{\prime} \hookrightarrow^{i_{1}} B_{1} ;
$$

here $\left(L^{2}\left(\Omega, \omega_{0}, \mathbf{R}^{m}\right)\right)^{\prime} \equiv\left(L^{2}\left(\Omega, \omega_{0}, \mathbf{R}^{m}\right)\right)$ is the canonical isomorphism $\theta$ of the Hilbert space $L^{2}\left(\Omega, \omega_{0}, \mathbf{R}^{m}\right)$ and its, for $\imath: B_{0} \rightarrow B$ we take simply the injection mapping, and for $\jmath: B \rightarrow B_{1}$ we take the concatenation of injections and the canonical isomorphism given bay 4.2 i.e: $\jmath=\imath_{1} \circ \theta \circ \imath_{0}$. Then $\left(u_{k}\right)$ is a bounded sequence in $L^{p}\left(0, T, B_{0}\right)$ by $\left(A_{1}\right)$. Next observe that $\left(\partial_{t} u_{k}\right)$ or rather $\left(\partial_{t} j o i\right.$ o $\left.u_{k}\right)$ is a bounded sequence in $\left(L^{p^{\prime}}\left(0, T,\left(W_{0}^{r, 2}\left(\Omega, \omega_{\alpha}, \mathbf{R}^{m}\right)\right)^{\prime}\right)\right)$ by $\left(A_{2}\right)$. Hence, from 4.1 , we may conclude that there exists a subsequence, which we still denote by $u_{k}$, having the property that the second part of 4.1 hold. Notice that in order to have the strong convergence simultaneously for all $r<p^{*}$, the usual diagonal sequence procedure applies finally, as $p>1$, the sequence converges in measure see, e-g. [7] (proposition 2-29). Which end the proof.

Lemma 4.2. (div-curl inequality) Suppose $\left(A_{1}-A_{6}\right)$ and assume ( after passing to a suitable subsequence if necessary) that $\left(D u_{k}\right)$ generates the young measure $\vartheta_{(x, t)}$. Then the following inequality hold:

$\int_{\Omega_{T}} \int_{\mathbf{M}^{m \times n}} \sigma(x, t, u, \lambda): \lambda d \vartheta_{(x, t)}(\lambda) d x d t \leq \int_{\Omega_{T}} \int_{\mathbf{M}^{m \times n}} \sigma(x, t, u, \lambda): D u d \vartheta_{(x, t)}(\lambda) d x d t$ $(4.3)$

Proof of lemma 4.2 Let us consider the sequence:

$I_{k}=\left(\sigma\left(x, t, u_{k}, D u_{k}\right):\left(D u_{k}-D u\right)\right)=\left(\sigma_{k}: D u_{k}\right)-\left(\sigma_{k}: D u\right)$.

By conditions $\left(A_{4}\right)$ and $\left(A_{5}\right)$, the negative part $I_{k}^{-}$of $I_{k}$ is equiintegrable. Hence, in view of $\left(A_{3}\right)$, we may use the (Fatou - Lemma ) which gives that $X=\liminf _{k \rightarrow \infty}\left(\int_{\Omega_{T}} I_{k} d x d t \geq \int_{\Omega_{T}} \int_{\mathbf{M}^{m \times n}} \sigma(x, t, u, \lambda):(\lambda-D u) d \vartheta_{(x, t)}(\lambda) d x d t\right.$ $X=\liminf _{k \rightarrow \infty}\left(\int_{\Omega_{T}} \sigma_{k}:\left(D u_{k}-D v_{k}\right) d x d t+\int_{\Omega_{T}} \sigma_{k}:\left(D v_{k}-D u\right) d x d t\right)$ $=\liminf _{k \rightarrow \infty}\left(\int_{\Omega_{T}} \sigma_{k}:\left(D v_{k}-D u\right) d x d t \leq c . \liminf _{k \rightarrow \infty}\left\|\sigma_{k}\right\|_{L^{p^{\prime}}\left(\Omega_{T}, \omega^{*}\right)} \| v_{k}-\right.$ $u \|_{L^{p}\left(0, T, W_{0}^{1, p}\left(\Omega, \omega, \mathbf{R}^{m}\right)\right)}$

$=0$ by the Hölder inequality and $\left(A_{4}\right)$. Thus the conclusion follows. 


\section{A second parabolic div-curl inequality}

We state here the second set of conditions:

- $\left(c_{1}\right): u_{k} \in L^{1}\left(0, T, W_{0}^{1, p}\left(\Omega, \omega, \mathbf{R}^{m}\right)\right)$ for all; $k \in \mathbf{N}$, and there exists some $c>0$ and $\rho>0$ such that:

$\sup _{k} \sum_{i j} \int_{\Omega_{T}}\left|D_{i j} u_{k}\right|^{\rho} \omega_{i j} d x d t \leq c$.

- $\left(c_{2}\right)$ : The sequence $u_{k}$ converges in measure to some function $u$ and $u(., t)$ is almost everywhere approximately differentiable a.e. $t \in] 0, T[$.

- $\left(c_{3}\right): \sigma: \Omega_{T} \times \mathbf{R}^{m} \times \mathbf{M}^{m \times n} \longrightarrow \mathbf{M}^{m \times n}$ and $\tau[e]: \Omega_{T} \times \mathbf{R}^{m} \times \mathbf{M}^{m \times n} \longrightarrow \mathbf{M}^{m \times n} ; e \in \mathbf{N}$ are Carathéodory conditions.

- $\left(c_{4}\right): \sigma$ and $\tau[e]$, satisfy all the angle structure conditions or satisfies all the sign structure conditions:

(Angle condition) For all $(x, t) \in \Omega_{T}, u \in \mathbf{R}^{m}$ and $M ; F \in \mathbf{M}^{m \times n}$, with $M$ of the form $I d-a \otimes a$ where $a \in \mathbf{R}^{m}$ satisfies $|a| \leq 1$; $\sigma(x, t, u, F): M F \geq 0$, for all matrix.

$M \in \mathbf{M}^{m \times m}$ of the form $M=I d-a \otimes a$ with $|a| \leq 1$.

(The sign condition ) For all $(x, t) \in \Omega_{T}, u \in \mathbf{R}^{m}$ and $F \in \mathbf{M}^{m \times n}$, we have

$\sigma_{j}(x, t, u, F): F_{j} \geq 0$, for all $1 \leq j \leq m$ with

$F_{j}$ and $\sigma_{j}$ are the cologne $\mathrm{j}$ of matrix $F$ and $\sigma$.(this conditions is verifies for $\tau[e]$ )

- $\left(c_{5}\right)$ : The sequence $\sigma_{k}(x, t)=\sigma\left(x, t, u_{k}, D u_{k}\right)$ is equiintegrable.

- $\left(c_{6}\right)$ : The sequence $\tau_{k}(x, t) \equiv \tau[k]\left(x, t, u_{k}, D u_{k}\right)$; and converges weakly to 0 in the space $L^{1}\left(\Omega_{T}, \mathbf{M}^{m \times n}\right)$.

- $\left(c_{7}\right)$ : The sequence $g_{k}=-\operatorname{div}\left(\sigma_{k}+g\left(x, t, u_{k}\right)+\tau_{k}\right)-v-f$ is bounded in $L^{1}\left(\Omega_{T}, \mathbf{R}^{m}\right)$.

- $\left(c_{8}\right): D u_{k} \in L_{l o c}^{i}\left(\Omega_{T}, \omega, \mathbf{M}^{m \times n}\right)$ and $\left(\sigma_{k}+g\left(x, t, u_{k}\right)+\tau_{k}\right) \in L_{l o c}^{i}\left(\Omega_{T}, \omega^{*}, \mathbf{M}^{m \times n}\right)$.

for some $i ; 1<i<\infty$.

Lemma 5.1. (Second div-curl inequality) Suppose $\left(C_{1}\right)-\left(C_{2}\right)$, and assure ( after passing to a suitable subsequence if necessary ) that $D u_{k}$ generates 
a Young measure $\vartheta_{(x, t)}$. Then (after passing to a subsequence), the $\sigma_{k}$ converges weakly in $L^{1}\left(\Omega_{T}, \mathbf{M}^{m \times n}\right)$ and the weak limit $\bar{\sigma}$ is given by: $\bar{\sigma}(x, t)=\left\langle\vartheta_{(x, t)} ; \sigma(x, t, u(x, t),).\right\rangle$, moreover the following inequality hold $\int_{\mathbf{M}_{T} \text {. }} \sigma(x, t, u(x, t), \lambda): \lambda d \vartheta_{(x, t)}(\lambda) \leq \bar{\sigma}(x, t): a p D u(x, t)$. for a.e. $(x, t) \in$

Proof of lemma 5.1 Proof of lemma is a slightly modification of the corresponding in lemma $(6-2-1)$ in [6] pages (54-61).

Remark 5.1. If the sequence $D u_{k}$ is equiintegrable then $D u(x, t)=a p D u(x, t)=\left\langle\vartheta_{(x, t)} ; i d\right\rangle$ almost everywhere. by the Ball's theorem.

The parabolic div-curl inequality will be the key ingredient to pass the limit in the different cases, but we need some additional information on the Young measure $\vartheta_{(x, t)}$ generated by the sequence of the gradients $D u_{K}$ to exploit lemma (div-curl inequality) these properties are the following:

- $\left(N_{1}\right): \vartheta_{x, t}$ is a probability measure for almost every $(x, t) \in \Omega_{T}$.

- $\left(N_{2}\right): \vartheta_{x, t}$ is a homogeneous $W^{1, P}$ - gradient young measure for almost every $(x, t) \in \Omega_{T}$.

- $\left(N_{3}\right): \vartheta_{x, t}$ satisfies $\left\langle\vartheta_{(x, t)}, i d\right\rangle=a p D u(x, t)$ almost everywhere in $\Omega_{T}$.

Note that these properties hold if the condition $\left(A_{1}\right)$ is fulfilled .

Lemma 5.2. Assume that the sequence $u_{k}$ satisfies $\left(A_{1}\right)$; then the Young measure $\vartheta_{(x, t)}$ generated by the sequence of the gradient $D u_{k}$ satisfies $\left(N_{1}\right)-\left(N_{3}\right)$.

\section{Proof of lemma 5.2}

Noting that $\left(A_{1}\right)$ implies that $a p D u(x, t)=D u(x, t)$, follows exactly as in [8] (prop 4-3). We mention that the properties, also hold if the conditions $\left(C_{1}\right)-\left(C_{2}\right)$ and a particular estimate are fulfilled as shown in [4] (lemma $9)$, with a slight modifications. 
Lemma 5.3. Assume that the sequence $\left(u_{k}\right)$ satisfies $\left(C_{1}\right)-\left(C_{2}\right)$ and $\sup _{k \in \mathbf{N}} \sum_{i, j} \int_{\left|u_{k}\right|<R}\left|D_{i j} u_{k}\right|^{\rho} \omega_{i j} d x d t \leq c$, for all $R>0$; for some $\rho>1$. Then the Young measure $\vartheta_{(x, t)}$ generated by the sequence of the gradients $D u_{k}$ satisfies $\left(N_{1}\right)-\left(N_{3}\right)$.

Proof of lemma 5.3 The proof of lemma is a slightly modification of the corresponding in lemma (11-3-2) in [6]

\section{Passage to the limit for $\sigma$}

Proposition 6.1. Suppose that $\left(A_{1}\right)-\left(A_{6}\right)$ or $\left(C_{1}\right)-\left(C_{8}\right)$ hold further assume that the Young measure $\vartheta_{(x, t)}$ generated by the gradients $D u_{k}$ satisfies $\left(N_{1}\right)-\left(N_{3}\right)$ and that one of the following conditions holds $(a)-(b)-(c)-(d)$ then(after passage to a subsequence ) the sequence $\sigma_{k}$ converges weakly in $L^{1}\left(\Omega_{T} ; \mathbf{M}^{m \times n}\right)$ and the weak limit $\bar{\sigma}$ is given by:

$\bar{\sigma}(x, t)=\sigma(x, t, u(x, t), D u(x, t))$. If $(b)-(c)$ or $(d)$ holds, then:

$\sigma\left(x, t, u_{k}, D u_{k}\right) \rightarrow \sigma(x, t, u, D u)$ in $L^{1}\left(\Omega_{T} ; \mathbf{M}^{m \times n}\right)$

In case $(c)$ and $(d)$; it follows in addition that $D u_{k} \rightarrow D u$ in measure and thus also almost everywhere in $\Omega_{T}$.

Before to prove proposition, we note that by proceeding exactly as in the elliptic case, we get the analogous result to lemma $(6-4-2)$ see [6] and $[1]$.

Lemma 6.1. Suppose that $\left(A_{1}\right)-\left(A_{6}\right)$ or $\left(C_{1}\right)-\left(C_{8}\right)$ hold. Further assume that $\vartheta_{(x, t)}$ is the Young measure generated by the gradients $D u_{k}$ and satisfies $\left(N_{1}\right)-\left(N_{3}\right)$. If the map $F \mapsto \sigma(x, t, u, F)$ is monotone for all $(x, t, u) \in \Omega \times] 0 ; T\left[\times \mathbf{R}^{m}\right.$ then:

$$
S p t \vartheta_{(x, t)} \subset\{\lambda /[\sigma(x, t, u, \lambda)-\sigma(x, t, u, D u)]:(\lambda-D u)=0\} .
$$

\section{Proof of lemma 6.1}

The proof of lemma is a slightly modification of the corresponding in lemma (11-4-2) see:[6]. 
Proof of proposition 6.1 We start with the easiest case:

Case (c): since $\sigma$ is monotone by assumption, 6.1 hold by lemma, by strict monotonicity, it follows from 6.1 that

$\vartheta_{(x, t)}=\delta_{D u(x, t)}$ for almost all $(x, t) \in \Omega_{T}$; and hence $D u_{k} \rightarrow D u$ in measure for $k \rightarrow \infty$ by proposition $(4-2-1)$ in [6], since we have already that $u_{k} \rightarrow u$ in measure for $k \rightarrow \infty$ by $\left(A_{1}\right) ;\left(A_{2}\right)$ and proposition 4.1 or by $\left(C_{2}\right)$ we may infer that (after extraction of a suitable subsequence, if necessary see: [7] (theorem, 2-30) $u_{k} \rightarrow u$ and $D u_{k} \rightarrow D u$ almost everywhere in $\Omega$ for $k \rightarrow \infty$.

thus, from the continuity condition $\left(A_{3}\right)$ or $\left(C_{3}\right)$ it follows that: $\sigma\left(x, t, u_{k}, D u_{k}\right) \rightarrow$ $\sigma(x, t, u, D u)$ almost everywhere in $\Omega$ for $k \rightarrow \infty$. Since, by assumption $\left(A_{4}\right)$ or $\left(A_{5}\right), \sigma_{k}(x, t)$ is equiintegrable, it follows from the Vitali's converge theorem $(1-2-1)$ in $[6]$

that; $\sigma\left(x, t, u_{k}, D u_{k}\right) \rightarrow \sigma(x, t, u, D u)$ in $L^{1}\left(\Omega_{T} ; \mathbf{M}^{m \times n}\right)$ which proves the proposition in this case.

Case (d): Assume that $\vartheta_{(x, t)}$ is not a Dirac mass on a set $(x, t) \in M \subset$ $\Omega_{T}$ of positive Lebesgue measure $|M|>0$. Then, by the strict p-quasimonotonicity of $\sigma(x, t, u,$.$) and \left(N_{2}\right)$, we have for a.e. $(x, t) \in M$ with: $\bar{\lambda}=\left\langle\vartheta_{(x, t)} ; i d\right\rangle=a p D u(x, t)$, by $\left(N_{3}\right)$ :

$$
\begin{aligned}
\int_{\mathbf{M}^{m \times n}} \sigma(x, t, u, \lambda): \lambda d \vartheta_{(x, t)}(\lambda) & >\int_{\mathbf{M}^{m \times n}} \sigma(x, t, u, \bar{\lambda}): \lambda d \vartheta_{(x, t)}(\lambda) \\
& -\int_{\mathbf{M}^{m \times n}} \sigma(x, t, u, \bar{\lambda}): \bar{\lambda} d \vartheta_{(x, t)}(\lambda) \\
& +\int_{\mathbf{M}^{m \times n}} \sigma(x, t, u, \lambda): \bar{\lambda} d \vartheta_{(x, t)}(\lambda) \\
=\int_{\mathbf{M}^{m \times n}} \sigma(x, t, u, \lambda): \bar{\lambda} d \vartheta_{(x, t)}(\lambda) &
\end{aligned}
$$

Where we used $\left(N_{1}\right)$, we claim now that we obtain a contradiction. Indeed, if $\left(C_{1}\right)-\left(C_{8}\right)$ hold, it follows by second div-curl inequality that:

$$
\begin{aligned}
\int_{\mathbf{M}^{m \times n}} \sigma(x, t, u, \lambda): \lambda d \vartheta_{(x, t)}(\lambda) & >\int_{\mathbf{M}^{m \times n}} \sigma(x, t, u, \lambda): \bar{\lambda} d \vartheta_{(x, t)}(\lambda) \\
& \geq \int_{\mathbf{M}^{m \times n}} \sigma(x, t, u, \lambda): \lambda d \vartheta_{(x, t)}(\lambda) .
\end{aligned}
$$

On the other hand if $\left(A_{1}\right)-\left(A_{6}\right)$ hold, by integrating 6.2 over $\Omega$ and using the div-curl inequality in lemma 4.2 , we get: 


$$
\begin{aligned}
\int_{\Omega_{T}} \int_{\mathbf{M}^{m \times n}} \sigma(x, t, u, \lambda): \lambda d \vartheta_{(x, t)}(\lambda) d x d t & >\int_{\Omega_{T}} \int_{\mathbf{M}^{m \times n}} \sigma(x, t, u, \lambda): \bar{\lambda} d \vartheta_{(x, t)}(\lambda) d x d t \\
& \geq \int_{\Omega_{T}} \int_{\mathbf{M}^{m \times n}} \sigma(x, t, u, \lambda): \lambda d \vartheta_{(x, t)}(\lambda) d x d t .
\end{aligned}
$$

As desired, hence, we have $\vartheta_{(x, t)}=\delta_{\lambda}=\delta_{D u(x, t)}$ for almost every $(x, t) \in \Omega_{T}$; thus, it follows again by proposition $(4-2-1)$ in [6] that $D u_{k} \rightarrow D u$ in measures, The reminder of the proof in this case is exactly as in case (c).

Case (b): We start by noting that for almost all $(x, t) \in \Omega_{T}$, the support of $\vartheta_{(x, t)}$ is contained in the set where $W$ agrees with the supporting hyperplane.

$L=\{(\lambda, W(x, t, u, \bar{\lambda})+\sigma(x, t, u, \lambda)):(\lambda-\bar{\lambda})\}$

in $\bar{\lambda}=a p D u(x, t)$ i.e, it may be shown see [9]. as in the proof of proposition 6.1 that $\operatorname{spt\vartheta }_{(x, t)} \subset K_{(x, t)}$ where.

$K_{(x, t)}=\left\{\left(\lambda \in \mathbf{M}^{m \times n} / W(x, t, u, \lambda)=W(x, t, u, \bar{\lambda})+\sigma(x, t, u, \bar{\lambda}):(\lambda-\bar{\lambda})\right\}\right.$.

By the convexity of $W$ we have:

$W(x, t, u, \lambda) \geq W(x, t, u, \bar{\lambda})+\sigma(x, t, u, \bar{\lambda}):(\lambda-\bar{\lambda})\}$.

For all $\lambda \in \mathbf{M}^{m \times n}$ and thus $L$ is a supporting hyper-plane for all $\lambda \in$ $K_{(x, t)}$. Since the mapping $\lambda \rightarrow W(x, t, u, \lambda)$ is by assumptions continuously differentiable we obtain:

$$
\sigma(x, t, u, \lambda)=\sigma(x, t, u, \bar{\lambda}), \text { for all } \lambda \in K_{(x, t)} \supset \operatorname{spt\vartheta }_{(x, t)}
$$

and thus:

$$
\bar{\sigma}(x, t) \equiv \int_{\mathbf{M}^{m \times n}} \sigma(x, t, u, \lambda) d \vartheta_{(x, t)}(\lambda)=\sigma(x, t, u, \bar{\lambda})
$$

Now consider the Carathéodory function:

$\psi(x, t, u, \rho)=|\sigma(x, t, u, \rho)-\bar{\sigma}(x, t)|$, the sequence:

$\psi_{k}(x, t)=\psi\left(x, t, u_{k}(x, t), \bar{\lambda}_{k}(x, t)\right)$ is equiintegrable and thus $\psi_{k} \rightarrow \bar{\psi}$ weakly in $L^{1}\left(\Omega_{T}\right)$ and the weak limit $\bar{\psi}$ is given by:

$\bar{\psi}(x, t)=\int_{\mathbf{R}^{m} \times \mathbf{M}^{m \times n}}|\sigma(x, t, \eta, \lambda)-\bar{\sigma}(x, t)| d \delta_{u(x, t)}(\eta) \otimes d \vartheta_{(x, t)}(\lambda)$

$=\int_{s p t} \vartheta_{(x, t)}|\sigma(x, t, u(x, t), \lambda)-\bar{\sigma}(x, t)| d \vartheta_{(x, t)}(\lambda)=0$ by 6.3 and 6.4. Since $\psi_{k} \geq 0$ it follows that $\psi_{k} \rightarrow 0$ strongly in $L^{1}\left(\Omega_{T}\right)$. This again to pass to the limit and the proof of the case (b)is finished.

Case (a): We claim that in this case for almost all $(x, t) \in \Omega \times] 0 ; T[$ the following identity hold for all $M \in \mathbf{M}^{m \times n}$ on the support of $\vartheta_{(x, t)}$ : 
$(6.5) \sigma(x, t, u, \lambda): M=\sigma(x, t, u, \bar{\lambda}): M+\left(\nabla_{F} \sigma(x, t, u, \bar{\lambda}) M\right):(\lambda-\bar{\lambda})$,

where $\nabla_{F}$ is the derivative with respect to the third variable of $\sigma$ and $\bar{\lambda}=a p D u(x, t)$.

Indeed, by the monotonicity of $\sigma$ we have for all $\tau \in \mathbf{R}$

$$
(\sigma(x, t, u, \lambda)-\sigma(x, t, u, \bar{\lambda}+\tau M)):(\lambda-\bar{\lambda}-\tau M) \geq 0,
$$

whence, by 6.1 ,

$-\sigma(x, t, u, \lambda):(\tau M) \geq \tau\left(\left(\nabla_{F} \sigma(x, t, u, \bar{\lambda}) M\right)(\lambda-\bar{\lambda})-\sigma(x, t, u, \bar{\lambda}): M\right)+$ $\circ(\tau)$.

The claim follows from this inequality since the sign of $\tau$ is arbitrary. Since the sequence $\sigma_{k}(x, t)$ is equiintegrable by $\left(A_{4}\right)$, its weak $L^{1}$-limit $\bar{\sigma}$ is given by

$$
\begin{aligned}
& \bar{\sigma}(x, t)=\int_{s p t \vartheta_{(x, t)}} \sigma(x, t, u, \lambda) d \vartheta_{(x, t)}(\lambda) \\
& =\int_{s p t \vartheta_{(x, t)}} \sigma(x, t, u, \bar{\lambda}) d \vartheta_{(x, t)}(\lambda)+\left(\nabla_{F} \sigma(x, t, u, \bar{\lambda})\right)^{t} \int_{s p t \vartheta_{(x, t)}}(\bar{\lambda}-\lambda) d \vartheta_{(x, t)}(\lambda) \\
& =\sigma(x, t, u, \bar{\lambda})
\end{aligned}
$$

where we used 6.5 in this calculation. This finishes the proof of the case (c) and hence of the proposition.

Remark 6.1. In case, (b), we remark, that the relation 6.4 already states that $\sigma(x, t, u, \bar{\lambda})$ is the weak $L^{1}$ - limit of $\sigma_{k}(x, t)$, which is enough to pass to the limit in an equation which must hold in the distributional sense. However, we wanted to point out that in this case, the convergence is even strong in $L^{1}\left(\Omega_{T}, \mathbf{M}^{m \times n}\right)$.

\section{Galerkin Scheme}

Lemma 7.1. Let $\Omega$ bounded domain in $\left.\left.\mathbf{R}^{n}, p \in\right] \frac{2 n}{n+2} ; 2\right]$ and $\omega_{0 j} \in L^{1}(\Omega)$, for all $j=1 ; 2 ; \ldots ; m$, then: $L^{2}\left(\Omega, \omega_{0}, \mathbf{R}^{m}\right) \hookrightarrow L^{p}\left(\Omega, \omega_{0}, \mathbf{R}^{m}\right)$.

Proof of lemma 7.1 The case $p=2$ is evident.

For case $p \in] \frac{2 n}{n+2} ; 2[$ :

Let $u \in L^{p}\left(\Omega, \omega_{0}, \mathbf{R}^{m}\right)$, we have:

$$
\int_{\Omega}\left|u_{j}\right|^{p} d x=\int_{\Omega}\left|u_{j}\right|^{p}\left(\omega_{0 j}\right)^{\frac{p}{2}+\frac{2-p}{2}} d x=\int_{\Omega}\left|u_{j}\right|^{p}\left(\omega_{0 j}\right)^{\frac{p}{2}}\left(\omega_{0 j}\right)^{\frac{2-p}{2}} d x
$$


We applied the Hölder inequality we have:

$$
\begin{gathered}
\int_{\Omega}\left|u_{j}\right|^{p}\left(\omega_{0 j}\right)^{\frac{p}{2}}\left(\omega_{0 j}\right)^{\frac{2-p}{2}} d x \leq\left(\int_{\Omega}\left|u_{j}\right|^{2} \omega_{0 j}\right)^{\frac{p}{2}}\left(\int_{\Omega} \omega_{0 j}\right)^{\frac{2-p}{2}} \\
\leq c_{j}\left(\int_{\Omega}\left|u_{j}\right|^{2} \omega_{0 j}\right)^{\frac{p}{2}} \\
\sum_{j} \int_{\Omega} \omega_{0 j}\left|u_{j}\right|^{p} d x \leq \max _{j}\left(c_{j}\right) \sum_{j}\left(\int_{\Omega}\left|u_{j}\right|^{2} \omega_{0 j}\right)^{\frac{p}{2}} d x \\
\|u\|_{L^{p}\left(\Omega, \omega_{0}\right)} \leq \max _{j}\left(c_{j}\right)\|u\|_{L^{2}\left(\Omega, \omega_{0}\right)}
\end{gathered}
$$

Lemma 7.2. Let $\Omega$ bounded domain in $\left.\left.\mathbf{R}^{n}, p \in\right] \frac{2 n}{n+2} ; 2\right]$ and $\omega_{i j} \in L^{1}(\Omega)$, for all $j=1 ; 2 ; \ldots ; m$, and $i=0 ; 1 ; \ldots . . ; n$. Then $\left(W_{0}^{r, 2}\left(\Omega, \omega_{\alpha}, \mathbf{R}^{m}\right) \hookrightarrow\right.$ $W_{0}^{1, p}\left(\Omega, \omega, \mathbf{R}^{m}\right)$,

for $r \geq 1+n\left(\frac{1}{2}-\frac{1}{P}\right)$.

Proof of lemma 7.2 (by the Hölder inequality, and 7.1 By the theorem $(1-3-1)$ in [6] and proceeding as [8] (section 4-2) we may choose a set $\left(\delta_{i}\right)_{i \in \mathbf{N}}$ of functions which is an orthogonal basis in $L^{2}\left(\Omega, \omega_{0}, \mathbf{R}^{m}\right)$ and at the some time an orthogonal basis in $W_{0}^{r, 2}\left(\Omega, \omega_{\alpha}, \mathbf{R}^{m}\right)$. In particular, $\delta_{(i)_{i \in \mathbf{N}}}$ is uniformly bounded in $W_{0}^{1, p}\left(\Omega, \omega, \mathbf{R}^{m}\right)$ and then: $\left\|\delta_{i}\right\|_{1, \omega, p}+\left\|\delta_{i}\right\|_{p^{*}} \leq c$ for all $i \in \mathbf{N}$.

We will need bellow the $L^{2}\left(\omega_{0}\right)$ - orthogonal projector $p_{k}: L^{2}\left(\Omega, \omega_{0}, \mathbf{R}^{m}\right) \rightarrow L^{2}\left(\Omega, \omega_{0}, \mathbf{R}^{m}\right)$ on to $\operatorname{spam}\left(\delta_{1}, \delta_{2}, \ldots, \delta_{k}\right)_{k \in \mathbf{N}}$ :

of course, the operator norm $\left\|p_{k}\right\|_{\Im\left(W_{0}^{r, 2}\left(\omega_{\alpha}\right), W_{0}^{r, 2}\left(\omega_{\alpha}\right)\right)}=1$.

(The Galerkin scheme): New we build our Galerkin approximations, we make the following ansate for approximating solution of

$(Q P S)_{\omega}, u_{k}(x, t)=\sum_{i=1}^{k} a_{k, i}(t) \delta_{i}(x)$. Where $a_{k, i}:[0 ; T) \rightarrow \mathbf{R}$ are supposed to be continuous bounded functions. such that $u_{k}$ satisfies the boundary condition in $(Q P S)_{\omega}$ by constructions in the sense that $u_{0} \in$ $L^{p}\left(0, T, W_{0}^{1, p}\left(\Omega, \omega, \mathbf{R}^{m}\right)\right)$, we take core of the initial conditions in $(Q P S)_{\omega}$ by choosing the initial coefficients:

$a_{k, i}(0)=\left\langle u_{0}, \delta_{i}\right\rangle_{2, \omega_{0}}$ such that:

$u_{k}(., 0)=\sum_{i=1}^{k} a_{k, i}(0) \delta_{i}(.) \rightarrow u_{0}$, with $u_{0} \in L^{2}\left(\Omega, \omega_{0}, \mathbf{R}^{m}\right)$.

we try to determine the coefficients $a_{k, i}(t)$ in such that a way, that for every $k \in \mathbf{N}$ the system of ordinary differential equations

$\left\langle\partial_{t} u_{k}, \delta_{j}\right\rangle_{2, \omega_{0}}+\int_{\Omega} \sigma\left(x, t, u_{k}, D u_{k}\right): D \delta_{j} d x$ 
$=\left\langle v(t), \delta_{j}\right\rangle+\int_{\Omega} f\left(x, t, u_{k}\right) \delta_{j} d x-\int_{\Omega} g\left(x, t, u_{k}\right): D \delta_{j} d x$ for $j \in\{1 ; 2 ; \ldots ; k\}$.

Now we fix to $k \in \mathbf{N}$ for the moment. Let $0<\varepsilon<T$ and $J=[0 ; \varepsilon]$. Moreover we chose $r>0$ large enough; such that the set $B_{r}(0) \subset \mathbf{R}^{k}$ contains the vector $\left(a_{1 k}(0), \ldots ., a_{k k}(0)\right)$, and we set $k=\overline{B_{r}(0)}$. Then we have the following result.

Lemma 7.3. The application: $F: J \times k \mapsto \mathbf{R}^{k}$ given by:

$\left(t, a_{1}, \ldots . a_{k}\right) \mapsto\left(\left\langle v(t), \delta_{j}\right\rangle+\int_{\Omega} f\left(x, t, \Sigma_{i=1}^{k} a_{i} \delta_{i}\right) \delta_{j} d x-\int_{\Omega} g\left(x, t, \Sigma_{i=1}^{k} a_{i} \delta_{i}\right):\right.$

$\left.D \delta_{j} d x-\int_{\Omega}\left(\sigma\left(x, t, \Sigma_{i=1}^{k} a_{i} \delta_{i}, \Sigma_{i=1}^{k} a_{i} D \delta_{i}\right): D \delta_{j}\right) d x\right)_{j=1 ; 2 ; \ldots k}$

is a Carathéodory function. Moreover, each components $F_{j}$ may be estimated on $J \times k$ by

$$
\left|F_{j}\left(t, a_{1}, \ldots . . a_{k}\right)\right| \leq C(r, k) \cdot M(t)
$$

uniformly on $J \times k$, where $C(r, k)$ is a constant which depends on $r$ and $k$. And here $M$ is a functions dependent of $j ; k$ and $r$ belonging to $L^{1}(J)$.

Proof of lemma 7.3 The first claim follows from the condition $\left(P_{1}\right)$.

For the second claim; we note that the generalized Hölder inequality implies that:

$\left|I_{j}\right|=\left|\left\langle v(t), \delta_{j}\right\rangle\right| \leq\|v(t)\|_{-1, p^{\prime} ; \omega^{*}}\left\|\delta_{j}\right\|_{1, p ; \omega}$.

Moreover, on the one hand, if; $I I_{j}=\int_{\Omega}\left(\sigma\left(x, t, \Sigma_{i=1}^{k} a_{i} \delta_{i}, \Sigma_{i=1}^{k} a_{i} D \delta_{i}\right)\right.$ : $\left.D \delta_{j}\right) d x$, by the growth condition in $\left(P_{2}\right)$ and the Hölder inequality

$\left|I I_{j}\right| \leq \beta\left[\left\|\lambda_{1}(t)\right\|_{p^{\prime}}+c_{1}\left\|\sum_{i=1}^{k} a_{i} \delta_{j}\right\|_{1, p, \gamma}^{\frac{q}{p^{\prime}}}+c_{2}\left\|\sum_{i=1}^{k} a_{i} D \delta_{j} u\right\|_{1, p, \omega}^{p-1}\right] \|$ $D \delta_{j} \|_{1, p, \omega}$

$\leq \beta \max \left(1, c_{1}, c_{2}\right) \cdot\left[\left\|\lambda_{1}\right\|_{p^{\prime}}+\left\|\sum_{i=1}^{k} a_{i} \delta_{j}\right\|_{1, p, \gamma}^{\frac{q}{p^{\prime}}}+\left\|\sum_{i=1}^{k} a_{i} D \delta_{j} u\right\|_{1, p, \omega}^{p-1}\right] \|$ $D \delta_{j} \|_{1, p, \omega}$.

And by Hölder inequality we have:

$$
\begin{aligned}
& \left|I I I_{j}\right|=\left|\int_{\Omega} g\left(x, t, \Sigma_{i=1}^{k} a_{i} \delta_{i}\right): D \delta_{j} d x\right| \\
& \leq\left(\left\|b_{4}(t)\right\|_{p^{\prime}}+\left\|b_{5}(t)\right\|_{\frac{n}{p-1}}\left\|\Sigma_{i=1}^{k} a_{i} \delta_{i}\right\|_{p^{*}, \omega_{0}}^{\eta}\right)\left\|D \delta_{j}\right\|_{p, \omega} \\
& \left|I V_{j}\right|=\left|\int_{\Omega} f\left(x, t, \Sigma_{i=1}^{k} a_{i} \delta_{i}\right): \delta_{j} d x\right|
\end{aligned}
$$

$\leq\left\|b_{1}(t)\right\|_{p^{\prime}}\left\|\delta_{j}\right\|_{p, \omega_{0}}+\left\|b_{2}(t)\right\|_{\frac{n}{p}}\left\|\Sigma_{i=1}^{k} a_{i} \delta_{i}\right\|_{p^{*}, \omega_{0}}^{\gamma}\left\|\delta_{j}\right\|_{p, \omega_{0}}$ Then, in view of 
$\left\|\delta_{i}\right\|_{1, \omega, p}+\left\|\delta_{i}\right\|_{1, p^{*}, \omega} \leq c$ for all $i \in \mathbf{N}$ and since each variable $a_{i}$ satisfies: $\left|a_{i}\right| \leq r$; for all $i=1 ; \ldots . . ; k$ fora constant $r$, each component $F_{j}$ may be estimated in such a way that

$(7.2)\left|F_{j}\left(t, a_{1}, \ldots . . a_{k}\right)\right| \leq\left|I I_{j}\right|+\left|I_{j}\right|+\left|I I I_{j}\right|+\left|I V_{j}\right| \leq C(r, k) \cdot M(t)$

uniformly on $J \times K$, where $C(r, k)$ is a constant which depends on $r$ and $k$, and where $M(t) \in L^{1}(J)$ (independent of $j, k$ )and $r$ Then, in view of lemma 7.3 the Carathéodory existence result on ordinary differential equation applied to the system:

$$
\begin{aligned}
& a_{j}^{\prime}(t)=F_{j}\left(t, a_{1}(t), \ldots, a_{k}(t)\right) \\
& a_{j}(0)=a_{k, j}(0) ; j \in 1 ; \ldots ; k
\end{aligned}
$$

ensures existence of a distributional, continuous solutions $a_{j}$ (depending on $k$ ) of $7.3-7.4$ on a time interval $\left[0 ; \varepsilon^{\prime}\right.$ [ where $\varepsilon^{\prime}>0$, a priori, may depend on $k$. Moreover, the corresponding integral equation

$$
a_{j}(t)=a_{j}(0)+\int_{0}^{t} F_{j}\left(t, a_{1}(t), \ldots . . a_{k}(t)\right) d t
$$

holds on $\left[0 ; \varepsilon^{\prime}\left[\right.\right.$. Then, $u_{k}=\sum_{j=1}^{k} a_{j}(t) \delta_{j}$ is the desired (short time) solution of

$$
\begin{aligned}
& \left\langle\partial_{t} u_{k}, \delta_{k}\right\rangle_{2, \omega_{0}}+\int_{\Omega} \sigma\left(x, t, u_{k}, D u_{k}\right): D \delta_{k} d x \\
= & \int_{\Omega} v(x, t) \delta_{k} d x+\int_{\Omega} f\left(x, t, u_{k}\right) \cdot u_{k} d x-\int_{\Omega} g\left(x, t, u_{k}\right): D u_{k} d x
\end{aligned}
$$

with initial condition

$u_{k}(0)=\sum_{i=1}^{k} a_{k, i}(0) \delta_{i}(.) \rightarrow u_{0}$, with $u_{0} \in L^{2}\left(\Omega, \omega_{0}, \mathbf{R}^{m}\right)$. Now, we want to show that the local solution constructed above can be extended to the whole interval $[0 ; T$ [ independent of $k$, as a - word of warning we should mention, that the solution need not be unique.

Lemma 7.4. We have:

$A=\{t \in[0 ; T[:$ there exist a weak solutions of $7.3-7.4$ on $[0 ; T[\}=$ $[0 ; T[$. 
Proof of lemma 7.4 The first thing we want to establish is a uniform bound on the coefficients $\left(a_{k i}(t)\right)$. Since

$\left\langle\partial_{t} u_{k}, \delta_{k}\right\rangle_{2, \omega_{0}}+\int_{\Omega} \sigma\left(x, t, u_{k}, D u_{k}\right): D \delta_{k} d x$

$=\int_{\Omega} v(x, t) \delta_{k} d x+\int_{\Omega} f\left(x, t, u_{k}\right) \cdot u_{k} d x-\int_{\Omega} g\left(x, t, u_{k}\right): D u_{k} d x$.

is linear in $\delta_{k}$, it is allowed to use $u_{k}$ as a test function in precedent equation in place of $\delta_{k}$. This gives for a an arbitrary time $\tau$ in the existence interval $\int_{0}^{\tau}\left\langle\partial_{t} u_{k}, u_{k}\right\rangle_{2, \omega_{0}} d t+\int_{0}^{\tau} \int_{\Omega} \sigma\left(x, t, u_{k}, D u_{k}\right): D u_{k} d x d t$

$=\int_{0}^{\tau}\left\langle v(t), u_{k}\right\rangle d t+\int_{0}^{\tau} \int_{\Omega} f\left(x, t, u_{k}\right) \cdot u_{k} d x d t-\int_{0}^{\tau} \int_{\Omega} g\left(x, t, u_{k}\right): D u_{k} d x d t$.

For the first term we have:

$\int_{0}^{\tau}\left\langle\partial_{t} u_{k}, u_{k}\right\rangle_{2, \omega_{0}} d t=\sum_{j} \frac{1}{2} \int_{0}^{\tau} \partial_{t}\left(u_{k}^{j}\right)^{2} \omega_{0 j} d t$
$=\sum_{j} \frac{1}{2}\left\|u_{k}^{j}(\tau)\right\|_{2, \omega_{0}}^{2}-\sum_{j} \frac{1}{2}\left\|u_{k}^{j}(0)\right\|_{2, \omega_{0}}^{2} \geq \frac{1}{2}\left\|u_{k}(\tau)\right\|_{2, \omega_{0}}^{2}-c$

Since $u_{k}(., 0)$ converges in $L^{2}\left(\Omega, \omega_{0}, \mathbf{R}^{m}\right)$. Using the coercivity and the Hölder inequality in $\left(P_{2}\right)$ for the second term, we obtain:

$\int_{0}^{\tau} \int_{\Omega} \sigma\left(x, t, u_{k}, D u_{k}\right): D u_{k} d x d t \geq-\left\|\lambda_{2}\right\|_{L^{1}\left(\Omega_{T}\right)}-A^{\prime \alpha}\left\|\lambda_{3}\right\|_{L^{\left(\frac{p}{\alpha}\right)^{\prime}}\left(\Omega_{T}\right)} \|$
$u_{k}\left\|_{L^{p}\left(0, T, W_{0}^{1, p}\left(\Omega, \omega, \mathbf{R}^{m}\right)\right)}^{\alpha}+c_{2}\right\| u_{k} \|_{L^{p}\left(0, T, W_{0}^{1, p}\left(\Omega, \omega, \mathbf{R}^{m}\right)\right)}^{p}$.

By the the Hölder inequality for the third term we get

$\int_{0}^{\tau}\left\langle v(t), u_{k}\right\rangle d t \leq\|v\|_{L^{p^{\prime}}\left(0, T, W^{\left.-1, p^{\prime}(\Omega, \omega)\right)}\right.}\left\|u_{k}\right\|_{L^{p}\left(0, T, W_{0}^{1, p}(\Omega, \omega)\right)}$. Using the growth condition $\left(F_{1}\right)$ and the Hölder inequality for the fourth term, we obtain:

$\int_{0}^{\tau} \int_{\Omega} f\left(x, t, u_{k}\right) \cdot u_{k} d x d t$

$\leq\left\|b_{1}(t)\right\|_{L^{p^{\prime}}\left(\Omega_{T}\right)} \cdot\left\|u_{k}\right\|_{L^{p}\left(0, \tau, L^{p}\left(\Omega, \omega_{0}\right)\right)}+\int_{0}^{\tau}\left\|b_{2}(t)\right\|_{\frac{n}{p}} \cdot\left\|u_{k}\right\|_{p^{*}, \omega_{0}}^{\gamma+1} d t$.

$\leq A^{\prime}\left\|b_{1}\right\|_{L^{p^{\prime}}\left(\Omega_{T}\right)} \cdot\left\|u_{k}\right\|_{L^{p}\left(0, \tau, W_{0}^{1, p}(\Omega, \omega)\right)}+A^{\prime \gamma+1}\left\|b_{2}\right\|_{L^{1}\left(0, T, L^{\left.\frac{n}{p}(\Omega)\right)}\right.} \cdot \|$ $u_{k} \|_{L^{p}\left(0, \tau, W_{0}^{1, p}(\Omega, \omega)\right)}^{\gamma+1}$.

Finally, using the growth condition $\left(G_{1}\right)$ for the last term, we obtain:

$\int_{0}^{\tau} \int_{\Omega} g\left(x, t, u_{k}\right) \cdot D u_{k} d x d t$ 
$\leq\left\|b_{4}(t)\right\|_{L^{p^{\prime}}\left(\Omega_{T}\right)} \cdot\left\|D u_{k}\right\|_{L^{p}\left(0, \tau, L^{p}(\Omega, \omega)\right)}+\int_{0}^{\tau}\left\|b_{5}(t)\right\|_{\frac{n}{p-1}} \cdot\left\|u_{k}\right\|_{p^{*}, \omega_{0}}^{\eta} \cdot \|$ $D u_{k} \|_{p, \omega} d t$

$\leq A^{\prime} .\left\|b_{4}\right\|_{L^{p^{\prime}}\left(\Omega_{T}\right)} \cdot\left\|u_{k}\right\|_{L^{p}\left(0, \tau, W_{0}^{1, p}(\Omega, \omega)\right)}+A^{\prime \eta}\left\|b_{5}\right\|_{L^{\infty}\left(0, \tau, L^{\frac{n}{p-1}}(\Omega)\right)} \cdot \|$ $u_{k} \|_{L^{p}\left(0, \tau, W_{0}^{1, p}(\Omega, \omega)\right)}^{\eta+1}$.

The combination of these five estimates gives:

$\frac{1}{2}\left\|u_{k}(\tau)\right\|_{2}^{2} \leq A^{\prime}\left(\left\|b_{4}\right\|_{L^{p^{\prime}}\left(\Omega_{T}\right)}+\left\|b_{1}\right\|_{L^{p^{\prime}}\left(\Omega_{T}\right)}+\|v\|_{L^{p^{\prime}\left(0, T, W^{-1, p^{\prime}}(\Omega, \omega)\right)}}\right) \|$

$u_{k} \|_{L^{p}\left(0, \tau, W_{0}^{1, p}(\Omega, \omega)\right)}+\left(\left\|\lambda_{3}\right\|_{L^{\left(\frac{p}{\alpha}\right)^{\prime}\left(\Omega_{T}\right)}}+A^{\prime \gamma+1}\left\|b_{2}\right\|_{L^{\gamma \mp}\left(0, T, L^{\left.\frac{n}{p}(\Omega)\right)}\right.}+A^{\prime \eta} \|\right.$

$\left.b_{5} \|_{L^{\infty}\left(0, \tau, L^{\frac{n}{p-1}}(\Omega)\right)}\right)\left\|u_{k}\right\|_{L^{p}\left(0, T, W_{0}^{1, p}\left(\Omega, \omega, \mathbf{R}^{m}\right)\right)}^{\kappa^{\prime}}+c+\left\|\lambda_{2}\right\|_{L^{1}\left(\Omega_{T}\right)}-c_{2} \|$

$u_{k} \|_{L^{p}\left(0, T, W_{0}^{1, p}\left(\Omega, \omega, \mathbf{R}^{m}\right)\right)}^{p}$

$\equiv \psi\left(\left\|u_{k}\right\|_{L^{p}\left(0, \tau, W_{0}^{1, p}\left(\Omega, \omega, \mathbf{R}^{m}\right)\right)}\right)$

where $\kappa^{\prime}=\max (\alpha, \gamma+1, \eta+1)$ Since $p>1$, and $c_{2}>0$ we have:

$$
\psi(y) \rightarrow-\infty ; y \rightarrow+\infty
$$

and so $\psi\left(\left\|u_{k}\right\|_{L^{p}\left(0, T, W_{0}^{1, p}\left(\Omega, \omega, \mathbf{R}^{m}\right)\right)}\right)$ is bounded, since $\psi$ is continuous and $\left\|u_{k}\right\|_{2}^{2} \geq 0$.

Thus, in view of the definition of $a_{k, i}$ we may conclude that:

$$
\left|a_{k, i}(\tau)\right|_{\mathbf{R}^{k}}^{2}=\left\|u_{k}(\tau)\right\|_{2}^{2} \leq \bar{c}
$$

for a constant $\bar{c}$ which is independent of $\tau$ and $k$. Now, we prove that $A$ is non empty, open and closed in $[0 ; T]$. First, $A$ is clearly non- empty since we proved local existence above. Moreover, let $t \in A$ and $0<\tau_{1}<\tau_{2}<T$. Then by 7.5 , we have:

$$
\begin{aligned}
& \left|a_{k, j}\left(\tau_{1}\right)-a_{k, j}\left(\tau_{2}\right)\right| \leq \int_{\tau_{1}}^{\tau_{2}}\left|F_{j}\left(\tau, a_{k, i}(\tau), \ldots . ., a_{k, k}(\tau)\right)\right| d \tau \\
& \leq C(\bar{c}, k) \int_{\tau_{1}}^{\tau_{2}}|M(\tau)| d \tau
\end{aligned}
$$

since $M \in L^{1}(0, T)$, this implies that $\tau \mapsto a_{k, j}(\tau)$ is uniformly continuous.Then, we can restart to solve

$$
\begin{aligned}
& \left\langle\partial_{t} u_{k}, \delta_{k}\right\rangle_{2, \omega_{0}}+\int_{\Omega} \sigma\left(x, t, u_{k}, D u_{k}\right): D \delta_{k} d x \\
& =\int_{\Omega} v(x, t) \delta_{k} d x+\int_{\Omega} f\left(x, t, u_{k}\right) \cdot \delta_{k} d x-\int_{\Omega} g\left(x, t, u_{k}\right): D \delta_{k} d x
\end{aligned}
$$


for $k \in\{1 ; 2 ; \ldots ; m\}$.

At time $t$ with initial data $\lim _{\tau} \succ \longrightarrow T u_{k}(\tau)$ and hence get a solution of 7.3 7.4 on $[0, \tau+\varepsilon]$. This proves that $A$ is open. Finally, we consider a sequence $\tau_{i} \nearrow t ; \tau_{i} \in A$. Let $a_{k, j, i}$ denote the solution of $7.3-7.4$ we constructed on $\left[0, \tau_{i}\right]$ and define:

$$
a_{k, j, i} \equiv\left\{\begin{array}{cc}
a_{k, j, i}(\tau) & \text { if } \\
a_{k, j, i}\left(\tau_{i}\right) & \text { if } \left.\tau \in] \tau_{i} ; t\right] .
\end{array} \quad \tau \in\left[0 ; \tau_{i}\right]\right.
$$

The sequence $\left(\tilde{a_{k, j, i}}\right)_{i}$ is bounded and discontinuous on [0; $t$, as seen above. Hence, by the Arsela-Ascoli Theorem, a subsequence (again denoted by $a_{k_{j, j}, i}(\tau)$ converges uniformly in $\tau$ on $\left[0 ; t\left[\right.\right.$ to a continuous functions $\left.a_{k, j}(\tau)\right)$. Using Lebesgues convergence theorem in 7.5 it is now easy to see that $a_{k, j}(\tau)$ solves 7.3 on $[0 ; t[$. Hence $t \in A$ and thus $A$ is indeed closed, and as claimed, it follows $A=[0 ; T[$.

Proposition 7.1. The sequence of the Galerkin approximations $\left(u_{k}\right)$ is bounded in

$\left.L^{\infty}\left(0, T, L^{2}\left(\Omega, \omega_{0}, \mathbf{R}^{m}\right)\right) \cap L^{p}\left(0, T, W_{0}^{1, p}\left(\Omega, \omega, \mathbf{R}^{m}\right)\right)\right)$ and satisfies $\left(A_{1}\right)$ and $\left(A_{2}\right)$.

Proof of proposition 7.1 By testing equation:

$\left\langle\partial_{t} u_{k}, \delta_{j}\right\rangle_{2, \omega_{0}}+\int_{\Omega} \sigma\left(x, t, u_{k}, D u_{k}\right): D \delta_{j} d x$

$=\left\langle v(t), \delta_{j}\right\rangle+\int_{\Omega} f\left(x, t, u_{k}\right) \cdot \delta_{j} d x-\int_{\Omega} g\left(x, t, u_{k}\right): D \delta_{j} d x$

for: $j \in\{1,2, \ldots, k\}$. With $u_{k}$; we obtained in the proof of lemma 7.4 that 7.7 holds for arbitrary time $\tau \in\left[0 ; T\left[\right.\right.$. So $\left(u_{k}\right)$ is bounded in the space $L^{\infty}\left(0, T, L^{2}\left(\Omega, \mathbf{R}^{m}\right)\right)$. Moreover we have proved that $\psi\left(\left\|u_{k}\right\|_{L^{p}\left(0, T, W_{0}^{1, p}\left(\Omega, \omega, \mathbf{R}^{m}\right)\right)}\right)$ is positive, bounded and satisfies 7.6. Then there exists a constant $c>0$ such that

$$
\left\|u_{k}\right\|_{L^{p}\left(0, T, W_{0}^{1, p}\left(\Omega, \omega, \mathbf{R}^{m}\right)\right)} \leq c ; \quad \forall k \in \mathbf{N}
$$

Therefore, by extracting a suitable subsequence which is again denoted by $u_{k}$, we may assume by the Elberlein-Smuljan see e.g, [15] ( Page 141), that 
$u_{k} \rightarrow^{*} u$ in $L^{\infty}\left(0, T, L^{2}\left(\Omega, \omega_{0}, \mathbf{R}^{m}\right)\right)$

$u_{k} \rightarrow u$ in $L^{p}\left(0, T, W_{0}^{1, p}\left(\Omega, \omega, \mathbf{R}^{m}\right)\right)$

which proves in particular $\left(A_{1}\right)$. To verify $\left(A_{2}\right)$, observe that the time derivative

$\frac{d}{d t}\left(j \circ i \circ u_{k}\right):\left[0 ; T\left[\rightarrow B_{1}=\left(W_{0}^{s, 2}\left(\Omega, \omega_{\alpha}, \mathbf{R}^{m}\right)\right)\right)^{\prime}\right.$ is according to

$\left\langle\partial_{t} u_{k}, \delta_{j}\right\rangle_{2, \omega_{0}}+\int_{\Omega} \sigma\left(x, t, u_{k}, D u_{k}\right): D \delta_{j} d x$

$=\left\langle v(t), \delta_{j}\right\rangle+\int_{\Omega} f\left(x, t, u_{k}\right) \cdot \delta_{j} d x-\int_{\Omega} g\left(x, t, u_{k}\right): D \delta_{j} d x$

for: $j \in\{1 ; 2 ; \ldots ; k\}$. Given by: we recall that the projection operators $P_{k}$ are self adjoint with respect to the $L^{2}$ inner product.

$t \mapsto\left(\phi \mapsto\left\langle v(t), P_{k} \phi\right\rangle-\int_{\Omega} \sigma\left(x, t, u_{k}, D u_{k}\right): D\left(P_{k} \phi\right) d x+\int_{\Omega} f\left(x, t, u_{k}\right) \cdot\left(P_{k} \phi\right) d x-\right.$

$\left.\int_{\Omega} g\left(x, t, u_{k}\right): D\left(P_{k} \phi\right) d x\right)$. Now we have:

$\left\langle\frac{d}{d t}\left(\jmath \circ \imath \circ u_{k}\right)(t), \phi\right\rangle=I+I I+I I I+I V$ where

$I=\int_{0}^{T}\left\langle v(t), P_{k} \phi\right\rangle d t \leq\|v\|_{L^{p^{\prime}\left(0, T,\left(W^{-1, p^{\prime}}\left(\Omega, \omega^{*}\right)\right)\right.}}\left\|P_{k} \phi\right\|_{L^{p}\left(0, T,\left(W^{1, p}(\Omega, \omega)\right)\right.}$ by the generalized Hölder inequality,

$I I=-\int_{0}^{T} \int_{\Omega} \sigma\left(x, t, u_{k}, D u_{k}\right): D\left(P_{k} \phi\right) d x d t$

$\leq\left(\left\|\lambda_{1}\right\|_{L^{p^{\prime}}\left(\Omega_{T}\right)}+C\left(c_{1}\right)\left\|u_{k}\right\|_{L^{p}\left(0, T, W_{0}^{1, p}(\Omega, \omega)\right.}^{p-1}\right)\left\|P_{k} \phi\right\|_{L^{p}\left(0, T, W_{0}^{1, p}(\Omega, \omega)\right)}$ by the growth conditions in $\left(P_{2}\right)$ and the Hölder inequality.

$I I I=\int_{0}^{T} \int_{\Omega} f\left(x, t, u_{k}\right) \cdot\left(P_{k} \phi\right) d x d t$

$\leq\left(\left\|b_{1}\right\|_{L^{p^{\prime}\left(\Omega_{T}\right)}}+A^{\prime \gamma+1}\left\|b_{2}\right\|_{L^{\gamma \mp}\left(0, T, L^{\left.\frac{n}{p}(\Omega)\right)}\right.} \cdot\left\|u_{k}\right\|_{L^{p}\left(0, \tau, W_{0}^{1, p}(\Omega, \omega)\right)}^{\gamma}\right) . \|$

$P_{k} \phi \|_{L^{p}\left(0, \tau, W_{0}^{1, p}(\Omega, \omega)\right)}$.

By the growth condition $\left(F_{1}\right)$

$I V=-\int_{0}^{\tau} \int_{\Omega} g\left(x, t, u_{k}\right) \cdot D\left(P_{k} \phi\right) d x d t$

$\leq\left(\left\|b_{4}\right\|_{L^{p^{\prime}}\left(\Omega_{T}\right)}+A^{\prime \eta}\left\|b_{5}\right\|_{L^{\infty}\left(0, \tau, L^{\frac{n}{p-1}}(\Omega)\right)} \cdot\left\|u_{k}\right\|_{L^{p}\left(0, \tau, W_{0}^{1, p}(\Omega, \omega)\right)}^{\eta}\right) . \|$

$P_{k} \phi \|_{L^{p}\left(0, \tau, W_{0}^{1, p}(\Omega, \omega)\right)}$.

By the growth condition $\left(G_{1}\right)$. Since

$\left\|P_{k} \phi\right\|_{L^{p}\left(0, T, W_{0}^{1, p}(\Omega, \omega)\right)} \leq\left\|P_{k} \phi\right\|_{L^{p}\left(0, T, W_{0}^{r, 2}\left(\Omega, \omega_{\alpha}\right)\right)} \leq\|\phi\|_{L^{p}\left(0, T, W_{0}^{r, 2}(\Omega, \omega)\right)}$, the claim follows by the uniform bounded 7.8.

Remark 7.1. For further use, we note that from the bounded obtained for $\left\langle\frac{d}{d t}\left(\jmath \circ \imath \circ u_{k}\right)(t), \phi\right\rangle$ in the proof of proposition we can conclude that $\partial_{t} u$ 
(or rather $\left.\partial_{t}(\jmath \circ \imath \circ u)\right)$ is an element of the space $L^{p^{\prime}}\left(0, T,\left(W_{0}^{-1, p^{\prime}}\left(\Omega, \omega^{*}\right)\right)\right.$. This follows easily from the fact, that the set

$\left\{\phi \in L^{p}\left(0, T, W_{0}^{1, p}(\Omega, \omega)\right): \exists k \in \mathbf{N} / P_{k} \phi=\phi\right\}$ is dense in $L^{p}\left(0, T, W_{0}^{1, p}(\Omega, \omega)\right)$, as proved in [8] (Appendix II, with slight assimilation).

Remark 7.2. Recall that the space

$\left\{u \in L^{p}\left(0, T, W_{0}^{1, p}(\Omega, \omega)\right): \partial_{t}(\jmath \circ \imath \circ u) \in L^{p^{\prime}}\left(0, T,\left(W^{-1, p^{\prime}}\left(\Omega, \omega^{*}\right)\right.\right.\right.$ is continuously embedded in $C\left([0 ; T] ; L^{2}\left(\Omega, \omega_{0}\right)\right)$ see e-g [16] [ proposition 23-23], with slight assimilation.

Hence, we have that $u \in C^{0}\left([0 ; T] ; L^{2}\left(\Omega, \omega_{0}\right)\right)$ after possible modification of $u$ on a Lebesgue Zero-set of $[0 ; T]$. This gives $u(t,.) \in L^{2}\left(\Omega, \omega_{0}\right)$ a pointiest interpretation for all $t \in[0 ; T]$ and allows in particular to state that $u(t,$. attains its initial value

$$
u(., 0)=u_{0}
$$

continuously in $L^{2}\left(\Omega, \omega_{0}\right)$ see [8] (Appendix III). In the two following subsections, we prove that $u$ is really a solution of the system $(Q P S)_{\omega}$.

\section{Passage to the limit in $(\mathcal{Q P S})_{\omega}$}

In this subsection, we prove theorem 3.1. First, in order to apply proposition 7.1 , we verify that, under our assumptions, the conditions $\left(A_{1}\right)-\left(A_{6}\right)$ and $\left(N_{1}\right)-\left(N_{3}\right)$ holds for the Galerkin approximations solution $u_{k}$. $\left(A_{1}\right)$ and $\left(A_{2}\right)$ holds by proposition 7.1. Moreover, it follows then by lemma 7.2 that $\left(N_{1}\right)-\left(N_{3}\right)$ holds. The condition $\left(A_{3}\right)$ is equivalent to $\left(P_{1}\right)$. Next, to prove $\left(A_{4}\right)$, we note that by the growth condition in $\left(P_{2}\right)$ that

$$
\begin{aligned}
& \int_{\Omega_{T}}\left|\sigma_{r s}\left(x, t, u_{k}, D u_{k}\right)\right|^{p^{\prime}} \omega_{r s}^{*} d x d t \\
& \leq C \cdot\left(\int_{\Omega_{T}}\left|\lambda_{1}\right|^{p^{\prime}} d x d t+\sum_{j=1}^{m}\left|u_{k j}\right|^{q} \gamma_{j} d x d t+\sum_{i, j}\left|D_{i j} u_{k}\right|^{p} \omega_{i j} d x d t\right) ;
\end{aligned}
$$

with ( $c$ is a constants) which is uniformly bounded in $k$ by 7.8. To verify the equiintegrability of $\left(\sigma_{k}: D u_{k}\right)^{-}$, we fix an arbitrary measurable subset $\Omega^{\prime} \subset \Omega_{T}$. Then, on the one hand, the growth condition in $P_{2}$ implies that $\int_{\Omega^{\prime}}\left|\min \left(\sigma_{k}: D u_{k} ; 0\right)\right| d x d t \leq \int_{\Omega^{\prime}}\left|\lambda_{2}\right| d x d t+\int_{\Omega^{\prime}}\left|\lambda_{3}\right| \sum_{j=1}^{m} \omega_{1 j}^{\frac{\alpha}{p}}\left|u_{k j}\right|^{\alpha}$. by the Hölder inequality and 7.8. Thus $\left(A_{5}\right)$ follows. Finally, we prove that $\left(A_{6}\right)$ hold. According to Mazur's Theorem see e-g [15] (Theorem2, page $120)$, there exists a sequence $v_{k}$ in $L^{p}\left(0, T, W_{0}^{1, p}(\Omega, \omega)\right)$ where each $v_{k}$ is a 
convex linear combination of $u_{1}, \ldots, u_{k}$ such that $v_{k} \rightarrow u$ in $L^{p}\left(0, T, W_{0}^{1, p}(\Omega, \omega)\right)$. In particular, $v_{k}(t,.) \in \operatorname{span}\left(\delta_{1}, \delta_{2}, \ldots, \delta_{k}\right)$ for all $t \in[0, T]$. Now, we have

$$
\begin{aligned}
\int_{\Omega_{T}} \sigma_{k} & :\left(D u_{k}-D v_{k}\right) d x d t=\left\langle v, u_{k}-v_{k}\right\rangle-\sum_{j} \int_{\Omega_{T}}\left(u_{k}^{j}-v_{k}^{j}\right) \partial_{t}\left(u_{k}^{j}\right) \omega_{0 j} d x d t \\
& +\int_{\Omega_{T}} f\left(x, t, u_{k}\right) \cdot\left(u_{k}-v_{k}\right) d x d t-\int_{\Omega_{T}} g\left(x, t, u_{k}\right):\left(D u_{k}-D v_{k}\right) d x d t
\end{aligned}
$$

Observe that $\left(u_{k}-v_{k}\right) \in \operatorname{span}\left(\delta_{1}, \delta_{2}, \ldots, \delta_{k}\right)$ which allowed to use $\left\langle\partial_{t} u_{k}, \delta_{k}\right\rangle_{2}+\int_{\Omega} \sigma\left(x, t, u_{k}, D u_{k}\right): D \delta_{k} d x$ $=\int_{\Omega} v(x, t) \cdot \delta_{k} d x+\int_{\Omega} f\left(x, t, u_{k}\right) \cdot \delta_{k} d x d t-\int_{\Omega} g\left(x, t, u_{k}\right): D \delta_{k} d x d t$ in the equality above. The term $\left\langle v, u_{k}-v_{k}\right\rangle$ in 8.1 converge to zero, since $u_{k}-v_{k} \rightarrow$ 0 in $L^{p}\left(0, T, W_{0}^{1, p}(\Omega, \omega)\right)$ by the choice of $v_{k}$ and $\left(A_{1}\right)$.

Next, defining $I I_{k}=\int_{\Omega_{T}} f\left(x, t, u_{k}\right) \cdot\left(u_{k}-v_{k}\right) d x d t$, we infer by the Hölder inequality and the growth condition $\left(F_{1}\right)$ that

$$
\begin{aligned}
& \left|I I_{k}\right| \leq \int_{0}^{T}\left(\left\|b_{1}(t)\right\|_{p^{\prime}}\left\|u_{k}(t)-v_{k}(t)\right\|_{p, \omega_{0}}+\left\|b_{2}(t)\right\|_{\frac{n}{p}}\left\|u_{k}(t)\right\|_{p^{*}, \omega_{0}} \|\right. \\
& \left.u_{k}(t)-v_{k}(t) \|_{\frac{p^{*}}{p-\gamma}}\right) d t \\
& \left.\leq\left\|b_{1}\right\|_{L^{p^{\prime}}\left(\Omega_{T}\right)}\left\|u_{k}-v_{k}\right\|_{L^{p}\left(0, T, L^{p}\left(\Omega, \omega_{0}\right)\right)}\left\|u_{k}-v_{k}\right\|_{L^{p}\left(0, T, L^{p}\right.} \frac{p^{*}}{p-\gamma}(\Omega, \omega)\right) \\
& +\left\|b_{2}\right\|_{L^{\gamma^{\mp}}\left(0, T, L^{\frac{n}{p}}\right)}\left\|u_{k}\right\|_{L^{p}\left(0, T, L^{p^{*}}(\Omega, \omega)\right)}^{\gamma} \cdot
\end{aligned}
$$

Since the expression $\left\|u_{k}(t)\right\|_{p^{*}, \omega_{0}}$ is bounded and we have $u_{k}-v_{k} \rightarrow 0$ in $L^{p}\left(0, T, L^{r}\left(\Omega, \omega_{0}\right)\right)$ for all $r<p^{*}$ by the proposition 4.1 , the second term in 8.1 vanishes in the limit. For the third term in 8.1, we note that $g_{k}(x, t)=g\left(x, t, u_{k}\right) \rightarrow g(x, t, u)$ strongly in $L^{p^{\prime}}\left(\Omega_{T}\right)$ by $\left(G_{0}\right),\left(G_{1}\right)$ and proposition 4.1. To see this, we may assume by proposition 4.1 that $u_{k} \rightarrow u$ almost everywhere. Since by $\left(G_{1}\right),\left|g_{k}\right|^{p^{\prime}}$ is bounded by an integrals function, the claim follows from $\left(G_{0}\right)$ and the Dominated Convergence Theorem. We infer then that

$$
\begin{aligned}
& \left|\int_{\Omega_{T}} g_{k}:\left(D v_{k}-D u_{k}\right) d x d t\right| \leq\left\|g_{k}-g(., ., u)\right\|_{L^{p^{\prime}}\left(\Omega_{T}\right)}\left\|u_{k}-v_{k}\right\|_{L^{p}\left(0, T, W_{0}^{1, p}(\Omega, \omega)\right)} \\
& +\left|\int_{\Omega_{T}} g: D\left(v_{k}-u_{k}\right) d x d t\right| \rightarrow 0
\end{aligned}
$$


since $v_{k}-u_{k} \rightarrow 0$ in $L^{p}\left(0, T, W_{0}^{1, p}(\Omega, \omega)\right)$. Thus the third term in 8.1 disappears also.

Finally, for the last therm in 8.1, we have:

$$
\begin{array}{r}
-\sum_{j} \int_{\Omega_{T}}\left(u_{k}^{j}-v_{k}^{j}\right) \partial_{t}\left(u_{k}^{j}\right) \omega_{0 j} d x d t=-\sum_{j} \int_{\Omega_{T}} \frac{1}{2} \partial_{t}\left(u_{k}^{j}\right)^{2} \omega_{0 j} d x d t+\sum_{j} \int_{\Omega_{T}} v_{k}^{j} \partial_{t}\left(u_{k}\right) \omega_{0 j} d x d t \\
=-\frac{1}{2}\left\|u_{k}(T)\right\|_{2, \omega_{0}}^{2}+\frac{1}{2}\left\|u_{k}(0)\right\|_{2, \omega_{0}}^{2}+\sum_{j} \int_{\Omega_{T}} v_{k}^{j} \partial_{t}\left(u_{k}^{j}\right) \omega_{0 j} d x d t
\end{array}
$$

Concerning the last term in 8.1 we claim that for $k \rightarrow \infty$ we have:

$$
\sum_{j} \int_{\Omega_{T}} v_{k}^{j} \partial_{t}\left(u_{k}^{j}\right) \omega_{0 j} d x d t \rightarrow \sum_{j} \int_{\Omega_{T}} u^{j} \partial\left(u^{j}\right) \omega_{0 j} d x d t=\frac{1}{2}\|u(T)\|_{2, \omega_{0}}^{2}-\frac{1}{2}\left\|u_{0}\right\|_{2, \omega_{0}}^{2}
$$

To see this, let $\varepsilon>0$ be given. Then, there exists $M$ such that for all $l \geq m \geq M$ we have:

- $(i) \sum_{j}\left|\int_{\Omega_{T}}\left(u^{j}-v_{m}^{j}\right) \partial_{t}\left(u^{j}\right) \omega_{0 j} d x d t\right| \leq \varepsilon$. This is possible, since $\partial(j \circ$ $i \circ u) \in L^{p^{\prime}}\left(0, T, W_{0}^{-1, p^{\prime}}(\Omega, \omega)\right.$ and $v_{m} \rightarrow u$ in $L^{p}\left(0, T, W_{0}^{1, p}(\Omega, \omega)\right)$

- $(i i) \sum_{j}\left|\int_{\Omega_{T}}\left(v_{l}^{j}-v_{m}^{j}\right) \partial_{t}\left(u^{j}\right) \omega_{0 j} d x d t\right| \leq \varepsilon$. This is possible by the bound obtained for

$\left\langle\frac{d}{d t}\left(j \circ i \circ u_{k}\right)(t), \phi\right\rangle$ in the proof of proposition 7.1 since $\left(v_{l}-v_{k}\right) \in$ $\operatorname{span}\left(\delta_{1}, \delta_{2} \ldots \ldots, \delta_{k}\right)$ for all fixed $\left.t \in\right] 0, T[$.

Now, we fix $m \geq M$ and choose $m_{0} \geq m$ such that for all $l \geq m_{0}$;

$\sum_{j} \mid \int_{\Omega_{T}} v_{m}^{j}\left(\partial_{t}\left(u^{j}-u_{l}^{j}\right) \omega_{0 j} d x d t \mid \leq \varepsilon\right.$. This is possible, since $\partial_{t} u_{l} \rightarrow^{*} \partial_{t} u$ in $L^{p^{\prime}}\left(0, T,\left(W_{0}^{s, 2}\left(\Omega, \omega_{\alpha}\right)\right)^{\prime}\right)$. Combinations yields for all $l=l(\varepsilon) \geq m_{0}(\varepsilon)$

$\left|\sum_{j} \int_{\Omega_{T}} v_{l}^{j} \partial_{t}\left(u_{l}^{j}\right) \omega_{0 j} d x d t-\sum_{j} \int_{\Omega_{T}} u^{j} \partial_{t}\left(u^{j}\right) \omega_{0 j} d x d t\right|$

$\leq \sum_{j}\left|\int_{\Omega_{T}}\left(v_{l}^{j}-v_{m}^{j}\right) \partial_{t}\left(u_{l}^{j}\right) \omega_{0 j}\right| d x d t+\sum_{j}\left|\int_{\Omega_{T}} v_{m}^{j}\left(\partial_{t} u_{l}^{j}-\partial_{t} u^{j}\right) \omega_{0 j} d x d t\right|$

$+\sum_{j} \mid \int_{\Omega_{T}}\left(v_{m}^{j}-u^{j}\right) \partial_{t} u^{j} \omega_{0 j} d x d t \leq 3 \varepsilon$. 
This establishes 8.3. On the other hand, since $\left(u_{k}\right)_{k}$ is bounded in $L^{\infty}\left(0, T, L^{2}\left(\Omega, \omega_{0}\right)\right)$ by proposition 7.1 we have that $\left(u_{k}(T) \rightarrow u(T)\right)$ in $\left.L^{2}\left(\Omega, \omega_{0}\right)\right)$. Hence,

$$
\liminf _{k \rightarrow \infty}\left\|u_{k}(T)\right\|_{2, \omega_{0}} \geq\|u(T)\|_{2, \omega_{0}} .
$$

By construction of $u_{k}$ we also have

$$
\lim _{k \rightarrow \infty}\left\|u_{k}(0)\right\|_{\left.2, \omega_{0}\right)}=\left\|u_{0}\right\|_{2, \omega_{0}} .
$$

Using $8.5,8.4$ and 8.3 , in 8.2 , we conclude that $\left.\liminf _{k \rightarrow \infty}\left(-\sum_{j} \int_{\Omega_{T}}\left(u_{k}^{j}-v_{k}^{j}\right) \partial_{t}\left(u_{k}^{j}\right) \omega_{0}\right) d x d t\right) \leq 0$, thus $\left(A_{6}\right)$ is fulfilled.

So, $\left(A_{1}\right)-\left(A_{6}\right)$ and $\left(N_{1}\right)-\left(N_{3}\right)$ hold and we may infer from proposition 7.1 that $\sigma_{k}\left(x, t, u_{k}, D u_{k}\right)$ converge to $\sigma_{k}(x, t, u, D u)$ at least weakly in $L^{1}\left(\Omega_{T}, \mathbf{M}^{m \times n}\right)$, for $k \rightarrow \infty$. Since we have that $u_{k} \rightarrow u$ in measure on $\Omega_{T}$ by proposition 4.1, we may infer that $u_{k} \rightarrow u$ almost everywhere on $\Omega_{T}$, for $k \rightarrow \infty$.

Thus, for arbitrary $\varphi \in W_{0}^{1, p}\left(\Omega, \omega, \mathbf{R}^{m}\right)$, it follows from the continuity conditions $\left(F_{0}\right)$ and $\left(G_{0}\right)$ that $f\left(x, t, u_{k}\right) \cdot \varphi(x) \rightarrow f(x, t, u) \cdot \varphi(x)$ and $g\left(x, t, u_{k}\right) \cdot D \varphi(x) \rightarrow$ $g(x, t, u): D \varphi(x)$ almost everywhere on $\Omega_{T}$. Since, by the growth conditions in $\left(F_{1}\right)$ and $\left(G_{1}\right)$ and the uniform bound in proposition 7.1, $f\left(x, t, u_{k}\right) \cdot \varphi(x)$ and $g\left(x, t, u_{k}\right) \cdot D \varphi(x)$ are equiintegrable, it follows that

$f\left(x, t, u_{k}\right) \cdot \varphi(x) \rightarrow f(x, t, u) \cdot \varphi(x)$ and $g\left(x, t, u_{k}\right) \cdot D \varphi(x) \rightarrow g(x, t, u): D \varphi(x)$ in $L^{1}\left(\Omega_{T}\right)$ by Vitali's converge theorem.

Now, we take a test function $\varphi \in \cup_{i \in \mathbf{N}} \operatorname{span}\left(\delta_{1}, \delta_{2} \ldots \ldots ., \delta_{i}\right)$ and $\phi \in C_{0}^{\infty}([0, T])$ in

$\left\langle\partial_{t} u_{k}, \varphi(x)\right\rangle_{2, \omega_{0}}+\int_{\Omega} \sigma\left(x, t, u_{k}, D u_{k}\right): D \delta_{j} d x=\left\langle v(t), \delta_{j}\right\rangle+\int_{\Omega} f\left(x, t, u_{k}\right) \cdot \delta_{j} d x+$ $\int_{\Omega} g\left(x, t, u_{k}\right): D \delta_{j} d x$, for: $j \in\{1,2, \ldots, k\}$.

And integrate over the interval $] 0, T[$ and pass to the limit $k \rightarrow \infty$. The resulting equation is $\left.\sum_{j} \int_{\Omega_{T}} \partial_{t} u^{j}(x, t) \phi^{j}(t) \varphi(x) \omega_{0 j}\right) d x d t+\int_{\Omega_{T}} \sigma_{k}(x, t, u, D u): D \varphi(x) \phi(t) d x d t$ 
$=\langle v, \phi \varphi\rangle+\int_{\Omega_{T}} f(x, t, u) \cdot \varphi(x) \phi(t) d x d t+\int_{\Omega_{T}} g(x, t, u): D \varphi(x) \phi(t) d x d t$.

For arbitrary $\varphi \in \cup_{i \in \mathbf{N}} \operatorname{span}\left(\delta_{1}, \delta_{2}, \ldots, \delta_{i}\right)$ and $\phi \in C_{0}^{\infty}([0, T])$. By density of the linear span of these functions in $L^{p}\left(0, T, W_{0}^{1, p}(\Omega, \omega)\right)$, this proves that $u$ is in fact a weak solution of $(Q P S)_{\omega}$. Hence the theorem is proved.

\section{Examples}

Exemple 9.1. Let $\Omega$ is a bounded open domain in $\mathbf{R}^{n}$. In this example we suppose that the weighted functions: $\left(\omega_{i j}\right), 0 \leq i \leq n ; \quad 1 \leq j \leq m$, with $\omega_{i j}(x)=\omega(x), x \in \Omega$ for all $i ; j$ and $\omega(x)>0$ a.e. in $\Omega$. Hence we have the Hardy-Type inequalities in $\left(P_{0}\right)$ for all $u \in W_{0}^{1, p}(\Omega, \omega) ; q>p^{\prime}$.

Let $\sigma_{r s}(x, t, \eta, \xi)=\omega\left|\xi_{r s}\right|^{p-1} \operatorname{sign}\left(\xi_{r s}\right)$ for all $1 \leq r \leq n$ and $1 \leq s \leq m$; $\sigma=\left(\sigma_{r s}\right)_{r, s}$

$\sigma$ is a Carathéodory functions in $\left(P_{1}\right)$; $\sigma_{r s}$ verify $\left(P_{2}\right)-\left(P_{3}\right)$.

We take $d(x)=d(x, \partial \Omega), \omega_{0 j}(x)=d^{\lambda_{0}}(x)$ for all $1 \leq j \leq m, \omega(x)=$ $d^{\lambda}(x), \gamma(x)=d^{\mu}(x)$ we have: $W_{0}^{1, p}(\Omega, \omega) \hookrightarrow \hookrightarrow L^{q}(\Omega, \gamma)$ for $1<p \leq q<$ $\infty,($ resp $1 \leq q<p<\infty)$ if and only if $\lambda \neq p-1, \frac{n}{q}-\frac{n}{p}+1 \geq 0$ and $\frac{\mu}{q}-\frac{\lambda}{p}+\frac{n}{q}-\frac{n}{p}+1>0,\left(\right.$ resp $\lambda \in \mathbf{R}$, and $\left.\frac{\mu}{q}-\frac{\lambda}{p}+\frac{1}{q}-\frac{1}{p}+1>0\right)$. Hence the theorem 3.1 is proved.

Exemple 9.2. We suppose that $p=n=m=2 . \Omega=\left\{x \in \mathbf{R}^{2} ;|x|<1\right\}$ $\omega=\omega_{11}(x)=\omega_{22}(x)=d^{\lambda}(x)=\left|x-\frac{x}{|x|}\right|^{\lambda} \cdot \omega_{12} \equiv \omega_{21} \equiv 0$ and $\gamma(x)=$ $\left|x-\frac{x}{|x|}\right|^{\mu}$.

Let $\sigma_{r s}(x, t, \eta, \xi)=\omega\left|\xi_{r s}\right|^{p-1} \operatorname{sign}\left(\xi_{r s}\right)$ for all $1 \leq r \leq n$ and $1 \leq s \leq m$; $\sigma=\left(\sigma_{r s}\right)_{r, s}, f=g=0$. With $q>1 ; \lambda \neq p-1 ; \mu>\frac{q \lambda}{2}-2$, by this conditions the hypothesis $\left(P_{1}\right)-\left(P_{3}\right)$ are verify, thus the problem $(Q P S)_{\omega}$ has a solution.

\section{References}

[1] Y. Akdim, E. Azroul, and A. Benkirane, "Existence of solution for quasilinear degenerated elliptic equations", Electronic journal of differential equations, vol. 2001, Art ID. 71, Nov. 2001. [On line]. Available: https://bit.ly/2ykRfMj

[2] H. Brezis, Operateurs maximaux monotones et semi-groupes de contractions dans les espaces de Hilbert. Amsterdam: North-Holland, 1973. 
[3] F. Browder, "Existences theorems for nonlinear partial differential equations", in Global analysis, vol. 16, S.-S.- Chern and S. Smale, Eds. Providence, RI: American Mathematical Society, 1970, pp. 8-67, doi: $10.1090 / \mathrm{pspum} / 016$

[4] G. Dolzmann, N. Hungerbühler, and S. Müller, "Non-linear elliptic systems with measure-valued right hand side", Mathematische zeitschrift, vol. 226, no. 4, pp. 545-574, Dec. 1997, doi: 10.1007/PL00004354

[5] D. Blanchard and H. Redwane, "Existence of a solution for a class of parabolic equations with three unbounded nonlinearities, natural growth terms and L1 data", Arab journal of mathematical sciences, vol. 20, no. 2, pp. 157-176, Jul. 2014, doi: 10.1016/j.ajmsc.2013.06.002

[6] F. Augsburger, "Young measures and quasilinear systems in divergence form with weak monotonicity", PhD. thèse, Université de Fribourg, 2004.

[7] G. B. Folland, Real analysis: modern techniques and applications. New York, NY: Wiley, 1973.

[8] N. Hungerbühler, "Quasi-linear parabolic systems in divergence form with weak monotonicity", Duke mathematical journal, vol. 107, no. 3, pp. 497-520, Mar. 2001, doi:10.1215/S0012-7094-01-10733-3

[9] N. Hungerbüehler, Young measures and nonlinear PDEs. Birmingham, 1999. [On line] Available: https://bit.ly/3cL29d7

[10] J. Kristensen, "Lower semicontinuity in spaces of weakly differentiable functions", Mathematische annalen, vol. 313, no. 4, pp. 653-710, Apr. 1999. doi: $10.1007 / \mathrm{s} 002080050277$

[11] J.-L. Lions, Quelques methodes de resolution des problemes aux limites non lineaires. Paris: Dunod, 1969.

[12] J. Simon, "Compact sets in the spaceLp (0,T; B)", Annali di matematica pura ed applicata, vol. 146, no. 1, pp. 65-96, Dec. 1986, doi: 10.1007/BF01762360

[13] M. Valadier, "Young measures," in Methods of nonconvex analysis, vol. 1146, A. Cellina, Ed. Berlin: Springer, 1990, pp. 152-188, doi: $10.1007 / \mathrm{BFb} 0084935$

[14] M. Valadier, "A course on Young measures", Rendiconti dell'Istituto di Matematica dell'Università di Trieste, vol. 26 supp, pp. 349-394, 1994. [On line]. Available: https://bit.ly/3g8VNX7

[15] K. Yosida, Functional analysis, 6th ed. Berlin: Springer, 1980.

[16] E. Zeidler, Nonlinear functional analysis and its application, II/ A: linear monotone operators, New York, NY: Springer, 1990, doi: 10.1007/9781-4612-0985-0 